\title{
Single-single gravitational-wave captures in globular clusters: Eccentric deci-Hertz sources observable by DECIGO and Tian-Qin
}

\author{
Johan Samsing'1, Daniel J. D’Orazio ${ }^{2}$, Kyle Kremer ${ }^{3,4}$, Carl L. Rodriguez ${ }^{2}$, Abbas Askar ${ }^{5}$ \\ ${ }^{1}$ Niels Bohr International Academy, The Niels Bohr Institute, Blegdamsvej 17, 2100 Copenhagen, Denmark. \\ ${ }^{2}$ Department of Astronomy, Harvard University, 60 Garden Street Cambridge, MA 01238, USA. \\ ${ }^{3}$ Department of Physics and Astronomy, Northwestern University, \\ 2145 Sheridan Road, Evanston, IL 60208, USA. \\ ${ }^{4}$ Center for Interdisciplinary Exploration and Research in Astrophysics (CIERA), \\ 2145 Sheridan Road, Evanston, IL 60208, USA. \\ ${ }^{5}$ Lund Observatory, Department of Astronomy, and Theoretical Physics, \\ Lund University, Box 43, SE-221 00 Lund, Sweden.
}

\begin{abstract}
We study the formation rate of binary black hole mergers formed through gravitational-wave emission between unbound, single black holes in globular clusters. While the formation of these binaries in very dense systems such as galactic nuclei has been well studied, we show here that this process can operate in lower-density stellar systems as well, forming binaries at a rate similar to other proposed pathways for creating eccentric mergers. Recent advances in post-Newtonian cluster dynamics indicate that a large fraction of dynamically-assembled binary black holes merge inside their host clusters during weak and strong binary-single and binary-binary interactions, and that these systems may retain measurable eccentricities as they travel through the LIGO and LISA sensitivity bands. Using an analytic approach to modeling binary black holes from globular clusters, we show that the formation of merging binaries from previously unbound black holes can operate at a similar rate to mergers forming during strong binary encounters, and that these binaries inhabit a unique region of the gravitational-wave frequency space which can be identified by proposed deciHertz space-based detectors.
\end{abstract}

\section{INTRODUCTION}

Several binary black hole (BBH) mergers have now been observed by LIGO (the Laser Interferometer Gravitational Wave Observatory) and VIRGO, through their emission of gravitational waves (GWs) [1-7]. However, their astrophysical origin is still unknown, and the observed variety in both $\mathrm{BH}$ masses and spins [e.g. 8], in addition to the observed merger of binary neutron stars (NSs) [9], indicate that several formation mechanisms might be operating. Some of the recently proposed include: field binaries [10-23], stellar clusters [24-37], active galactic nuclei (AGN) discs [38-40], galactic nuclei (GN) [41-47], very massive stellar mergers [48-51], and single-single GW captures of primordial black holes [52$55]$.

From the GW signal of individual BBH merger events, one can measure the (redshifted) mass of the BHs, their spins [e.g. 56, 57], the BBH orbital eccentricity [e.g. 58$60]$, and even Doppler effects related to a possible movement of the BBH's center of mass (COM) [e.g. 61, 62] The question is; how can this information be used to distinguish the proposed astrophysical merger channels? Regarding BH spins, these are expected to be isotropically distributed for dynamically assembled BBH mergers, such as those forming in globular clusters (GCs), in contrast to those forming in isolation in the field [e.g. $56,63]$. In terms of eccentricity, a significant fraction of BBHs formed dynamically in clusters are expected to lead to a unique population of $\mathrm{GW}$ mergers that have measurable orbital eccentricities in bands from LISA (the
Laser Interferometer Space Antenna) [64-68] to LIGO [69-78], whereas field binaries are most likely to have fully circularized once observable. Although eccentric sources might form in other ways, e.g., through LidovKozai oscillations [e.g. 79-85], quadruple systems [e.g. 86], and single-single GW captures [e.g. 41, 53, 87, 88], eccentric BBH mergers forming in clusters are not only a natural outcome when BHs are present, but can also accurately be modeled using both simple analytical [e.g. 37, 75] and numerical techniques [e.g. 78]. This makes BBH orbital eccentricity a very promising parameter to use for constraining the cluster channel and its astrophysical properties. In addition to this, it has also been suggested that $\mathrm{BBH}$ populations in dense clusters can be probed through their interaction with nearby stars, e.g., through tidal interactions, which could result in electromagnetic observables [89-91].

In this paper we continue our studies on how BBH mergers form in stellar clusters, and in particular GCs; systems which recently have gained significant attention. One reason is that these systems are relatively easy to model as they stay more or less isolated for almost their entire life, during which they evolve through clean physical processes involving Newtonian and Post-Newtonian [e.g. 92] $N$-body dynamics. Recent developments in modeling this formation channel include how BBHs can form through strong binary-single interactions [e.g. 6976, 93, 94], weak binary-single interactions [e.g. 95, 96], strong binary-binary interactions [e.g. 77], and secular interactions [e.g. 97-99]. This recent work especially indicates that $\sim 50 \%$ of all BBHs assembled in GCs are likely to merge inside their cluster with a variety of GW 
peak frequencies and eccentricities [e.g. 78], which are tightly connected to the properties of their host cluster, whereas earlier prescriptions only resolved the dynamically ejected population [e.g. 24, 29]. These recent advances have major observational implications when considering the future of GW astrophysics where planned observatories such as the Einstein Telescope (ET) [e.g. 100], and the Cosmic Explorer (CE) [e.g. 101], will be able to map out every possible BBH merger in the entire visible Universe.

As one of the last pieces on how $\mathrm{BBH}$ mergers might form in GCs through few-body interactions (without the inclusion of a central massive $\mathrm{BH}$ ), we here describe the formation of single-single GW capture mergers [e.g. $88,102,103]$. Single-single GW captures have in the literature mainly been associated with GN hosting massive central BHs [e.g. 104]; however, recent work does indicate that single-single GW captures could operate at a nonnegligible rate in GCs partly due to their relative low velocity dispersion [e.g. 105]. In our present paper we build upon these earlier studies, and by the use of analytical arguments we not only prove that single-single GW captures in GCs lead to significant rates, but we also derive how single-single GW captures form compared to $\mathrm{BBH}$ mergers forming through the dominating three-body interaction channel inside the cluster. Especially, we find that the rate of single-single $\mathrm{GW}$ captures relative to the rate of GW mergers forming during binary-single interactions [e.g. 70], a population we loosely will refer to as binary-single GW mergers, does not strongly depend on neither the central velocity dispersion nor the density of the cluster, but mainly on the binary fraction and the shape of the BH population density profile. In most GCs the binary fraction is at the percent level [e.g. 78], for which we find that the rate of single-single GW captures leading to BBH mergers, should be similar to the rate of binary-single GW mergers.

The single-single GW capture mergers distribute differently across GW frequency and orbital eccentricity space compared to the other before mentioned few-body merger channels. This has been noticed before [105]; however, in our presented paper we derive for the first time the correct normalization of the single-single GW capture mergers compared to the mergers from binary-single interactions. This allow us to put forward a picture that unifies how BBHs distribute as a function of the binary fraction and the density profile of the single $\mathrm{BH}$ population. We show how the observable GW frequency and orbital eccentricity distributions change with these properties, which make us propose that single-single GW captures offer unique possibilities to probe the inner properties of BH subsystems [e.g. 106, 107].

Finally, from a combination of analytical and numerical techniques we illustrate how single-single GW capture mergers form right where the future planned GW observatories DECIGO [108, 109] and Tian Qin [110] are sensitive. As we clearly demonstrate in this paper, and also previously argued in [105], deci-Hertz observatories fill out a unique gap in GW frequency space that covers the range where the majority of dynamically assembled BBH mergers form, including those from single-single, binary-single and binary-binary interactions. Such observations will undoubtedly provide unique information about the distribution of BHs in dense stellar systems. This greatly adds to the astrophysical motivation to why such detectors should be built.

The paper is organized as follows. We start in Section II by calculating the cross sections for close two-body encounters originating from single-single and binary-single interactions, respectively. In Section III we convert our cross section expressions to formation rates, and estimate the rate of single-single GW capture mergers compared to binary-single GW mergers for two different cluster profiles. We then use simple Monte Carlo (MC) techniques in Section IV to derive GW peak frequency and eccentricity distributions for our considered dynamical $\mathrm{BBH}$ merger channels, and comment on the observational prospects. Our study is concluded in Section V.

\section{CROSS SECTIONS}

In this section we derive close encounter and GW inspiral merger cross sections for single-single and binarysingle interactions, respectively. These expressions are then used in Section III to derive absolute and relative merger rates relevant for a system like a GC. In all our calculations we assume the interacting $\mathrm{BHs}$ have the same mass $m$, which is a reasonable approximation for interactions happening in GCs due to mass segregation and frequent exchange interactions [e.g. 78, 111]. An illustration showing the interaction- and GW merger channels we consider in this paper is presented in Fig. 1.

In terms of notations, throughout the paper ' $G$ ' denotes Newton's constant, ' $c$ ' is the speed of light, and 'log' denotes the logarithm to the base 10 .

\section{A. Single-Single Interactions}

We start by considering the cross section for two single $\mathrm{BHs}$ to undergo an encounter with peri-center distance $r_{\mathrm{p}}$ less than some distance $R_{\mathrm{ss}}$. We denote this cross section by $\sigma_{\mathrm{ss}}^{<R}$, where ' $\mathrm{ss}$ ' is short for 'single-single'. In the gravitational focusing limit this cross section is given by [e.g. 70, 73],

$$
\sigma_{\mathrm{ss}}^{<R} \approx 2 \pi G \frac{2 m R_{\mathrm{ss}}}{v^{2}}
$$

where $m$ is the $\mathrm{BH}$ mass, and $v$ is the velocity dispersion of the $\mathrm{BH}$ subsystem (we do not distinguish between relative velocity and velocity dispersion in this paper).

For a single-single interaction to also result in a GW capture merger the energy radiated at the first peri-center passage from $\mathrm{GW}$ emission, $\Delta E_{\mathrm{GW}} \approx$ 


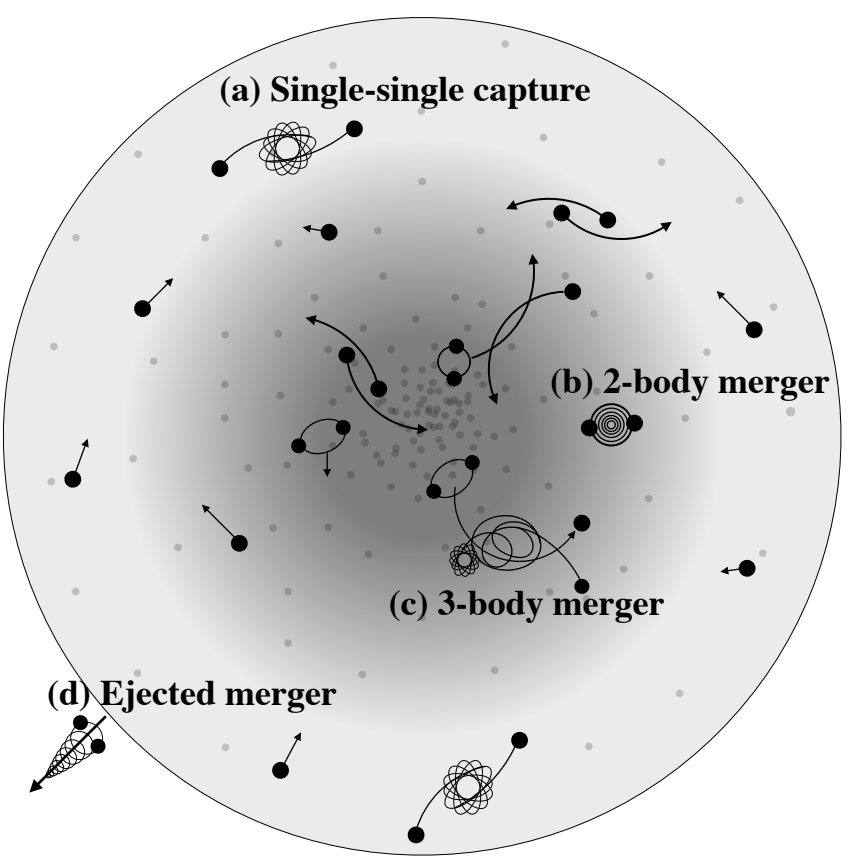

FIG. 1. Schematic overview of the different BBH merger types that we discuss in this paper. (a) Single-single capture: Two initially unbound $\mathrm{BHs}$ can become bound if they undergo a passage small enough for the energy radiated through GW emission to be larger than their initial orbital energy. This often results in a relatively prompt merger taking place inside the cluster, with a GW peak frequency near the DECIGO band. BBHs can also form through dynamical processes near the cluster core, and will after formation undergo primarily binary-single interactions, which result in at least the following three types of merger. (b) 2-body merger: If a BBH after a binary-single interaction survives with a SMA and eccentricity such that its GW inspiral time is less than its interaction time, then it will undergo what we refer to as a 2-body merger. The characteristic interaction time scale is $\sim 10^{7}$ years, which maps to a GW peak frequency near the LISA band. (c) 3-body merger: A BBH can also undergo a merger during a binary-single interaction if its peri-center distance is perturbed to a value small enough for the energy radiated over one orbit through GW emission is larger than the initial energy of the 3-body system. The time scale associated with this process is $\sim 1$ year, which maps to a GW peak frequency near the LIGO band. (d) Ejected merger: If the BBH does not undergo a 2-body or a 3-body merger inside its cluster, it will get dynamically ejected through a binary-single interaction. A large fraction of $\mathrm{BBH}$ s ejected in this way will merge outside the cluster within a Hubble time.

$(85 \pi / 12) G^{7 / 2} c^{-5} m^{9 / 2} r_{\mathrm{p}}{ }^{-7 / 2}$ (see [102]), must be greater than the initial energy between the two singles, $E_{\mathrm{ss}} \approx$ $(1 / 2) \mu v^{2}$, where $\mu$ is here the reduced mass [e.g. 70, 103]. The maximum peri-center distance from which a singlesingle GW capture can happen, denoted in this paper by $\mathcal{R}_{\mathrm{ss}}$, is the $r_{\mathrm{p}}$ that satisfies $\Delta E_{\mathrm{GW}}=E_{\mathrm{ss}}$, from which one finds,

$$
\mathcal{R}_{\mathrm{ss}}=\left(\frac{85 \pi}{24 \sqrt{2}}\right)^{2 / 7} \times \mathscr{R}_{\mathrm{m}}\left(\frac{c^{2}}{v^{2}}\right)^{2 / 7},
$$

where $\mathscr{R}_{\mathrm{m}}$ is the Schwarzschild radius of a $\mathrm{BH}$ with mass $m$. If one substitutes $R_{\mathrm{ss}}$ in Eq. (1) with $\mathcal{R}_{\mathrm{ss}}$ from the above Eq. (2) one gets the classical single-single GW capture cross section [103], that we here denote by $\sigma_{\mathrm{ss}}^{<\mathcal{R}}$. The value of $\mathcal{R}_{\mathrm{ss}}$ given above represents the theoretical upper limit for GW capture; however, the astrophysical upper limit is slightly smaller, and depends on local properties such as the BH density profile and velocity dispersion. The primary reason is that the definition of $\mathcal{R}_{\mathrm{ss}}$ given by Eq. (2) only ensures that the two single objects become bound after their first passage, but not that this newly formed binary actually undergoes a GW inspiral merger before it is interrupted by a later incoming object. We will take this correction into account later in our numerical experiments (see Eq. (34)), from which we find that this correction for a typical GC is not important. We therefore keep $\mathcal{R}_{\mathrm{ss}}$ as shown in Eq. (2) throughout our analytical sections.

\section{B. Binary-Single Interactions}

We now describe the derivation of the cross section for a binary-single interaction to result in two of the three objects to undergo an encounter with peri-center distance $r_{\mathrm{p}}$ less than some characteristic distance $R_{\mathrm{bs}}$. We denote this cross section $\sigma_{\mathrm{bs}}^{<R}$, where 'bs' is short for 'binarysingle'. For this, we start by expressing $\sigma_{\mathrm{bs}}^{<R}$ as the following product,

$$
\sigma_{\mathrm{bs}}^{<R}=\sigma_{\mathrm{bs}} \times P_{\mathrm{bs}}^{<R},
$$

where $\sigma_{\text {bs }}$ is the cross section for a binary to undergo a strong interaction with a single, and $P_{\mathrm{bs}}^{<R}$ denotes the probability for a strong binary-single interaction to result in two of the three objects to undergo an encounter with $r_{\mathrm{p}}<R_{\mathrm{bs}}$. The cross section $\sigma_{\mathrm{bs}}$ is in the gravitational focusing limit given by [73, 75],

$$
\sigma_{\mathrm{bs}} \approx 2 \pi G \frac{3 m a}{v^{2}},
$$

where $a$ is the semi-major axis (SMA) of the target binary. To derive $P_{\mathrm{bs}}^{<R}$ we make use of the formalism described in $[64,70,75,90]$. In short, the approach is to split up the chaotic binary-single interaction into a series of temporary metastable binary-single states, referred to here as intermediate states (IMSs). Each of these $\mathcal{N}$ states can be described by a binary with a bound single. The eccentricity distribution of the IMS binaries follows approximately that of a so-called thermal distribution, $P(e)=2 e$ [112], where the SMA is approximately equal to the SMA of the initial target binary, $a$. From this 
follows that the probability for a single IMS binary to have a peri-center distance $r_{\mathrm{p}}<R_{\mathrm{bs}}$ is $\approx 2 R_{\mathrm{bs}} / a$ [75]. As a binary-single interaction on average assembles $\mathcal{N}$ such IMS binaries during its chaotic evolution, the total probability $P_{\mathrm{bs}}^{<R}$ is simply given by,

$$
P_{\mathrm{bs}}^{<R} \approx \frac{2 R_{\mathrm{bs}}}{a} \mathcal{N}
$$

Using these relations, one now finds that the cross section $\sigma_{\mathrm{bs}}^{<R}$ can be written as,

$$
\sigma_{\mathrm{bs}}^{<R} \approx 2 \pi G \frac{6 m R_{\mathrm{bs}}}{v^{2}} \mathcal{N}
$$

From this it is clear that the cross section for a binarysingle interaction to result in a close encounter between two of the three objects with $r_{\mathrm{p}}<R_{\mathrm{bs}}$ is $\propto R_{\mathrm{bs}}$ and independent of the binary SMA $a$.

As in the single-single case, not all distances $R_{\mathrm{bs}}$ in the binary-single problem will lead to a GW inspiral merger. However, in contrast to the single-single case, defining a unique distance for GW inspiral merger in the binarysingle problem is not possible, as the distance from which a GW inspiral is possible changes for each IMS binary depending on the orbital period of the remaining bound single [70]. Therefore, one should in principle work with a distribution of GW inspiral distances; however, this has its own problems, and is therefore out of scope of this paper. Instead, to simplify our analysis and make it analytically tractable, we will in this paper work with a single characteristic value for the binary-single GW merger distance, that we denote by $\mathcal{R}_{\mathrm{bs}}$. Note here that this assumption still conserves all the right scaling properties of the problem [75]. Following [75], a reasonable value for $\mathcal{R}_{\mathrm{bs}}$ is the one for which the GW energy loss integrated over one peri-center passage $\Delta E_{\mathrm{GW}}$ (see Section II A) for two of the three objects equals the total initial energy of the three-body system $E_{\mathrm{bs}}$, that here is $\approx G m^{2} /(2 a)$. Note here that this requirement is exactly the same as in the single-single case, but with the energy of the initial binary-single system instead of the energy of the initial single-single system. Now solving for the peri-center distance $r_{\mathrm{p}}$ for which $\Delta E_{\mathrm{GW}}=E_{\mathrm{bs}}$, one finds,

$$
\mathcal{R}_{\mathrm{bs}} \approx\left(\frac{85 \pi}{24 \sqrt{2}}\right)^{2 / 7} \times \mathscr{R}_{\mathrm{m}}\left(\frac{a}{\mathscr{R}_{\mathrm{m}}}\right)^{2 / 7} .
$$

Substituting this expression for $\mathcal{R}_{\mathrm{bs}}$ into Eq. (6) results in the cross section for the formation of a GW inspiral that merges during the binary-single interaction; a cross section we denote $\sigma_{\mathrm{bs}}^{<\mathcal{R}}$. As first noted by [70], the cross section $\sigma_{\mathrm{bs}}^{<\mathcal{R}} \propto a^{2 / 7}$, which means that it increases with the initial SMA $a$. For a thorough discussion on the subject of binary-single interactions with dissipative terms (GW emission, tidal dissipation, etc.) see [e.g. 73, 93, 94]. Finally, as for the single-single GW captures, binary-single interactions and GW inspirals forming during such interactions can in principle be broken up be- fore completion by incoming objects [113]. However, this rarely happens for the systems we consider, and this 'infrared' correction will therefore not be discussed further in this paper.

\section{Comparing Binary-Single and Single-Single}

One of the key questions we explore in this paper is how important single-single GW capture mergers are compared to GW inspiral mergers forming during binarysingle interactions. To gain insight into this, we start by considering the following cross section ratio,

$$
\frac{\sigma_{\mathrm{bs}}^{<R}}{\sigma_{\mathrm{ss}}^{<R}}=3 \mathcal{N}\left(R_{\mathrm{bs}} / R_{\mathrm{ss}}\right) \approx 60\left(R_{\mathrm{bs}} / R_{\mathrm{ss}}\right),
$$

which follows from the use of Eq. (6) and Eq. (1). Note here that for the last equality we have used a value of $\mathcal{N}=20$, which follows from numerical experiments [75]. From this ratio we conclude that a $\mathrm{BBH}$ is about $3 \mathcal{N} \approx$ 60 times more 'effective' in forming two-body encounters with $r_{\mathrm{p}}<R_{\mathrm{ss}}=R_{\mathrm{bs}}$ compared to a single BH. Note here that this efficiency does not depend on any properties of the cluster or the $\mathrm{BBH}$ orbital parameters.

Two values of $R_{\mathrm{bs}}$ and $R_{\mathrm{ss}}$ that are particular interesting to compare are the distances from which a GW merger can form. By the use of Eq. (7) and Eq. (2) we find that,

$$
\frac{\mathcal{R}_{\mathrm{bs}}}{\mathcal{R}_{\mathrm{ss}}} \approx\left(\frac{a}{\mathscr{R}_{\mathrm{m}}} \frac{v^{2}}{c^{2}}\right)^{2 / 7}=\left(\frac{v}{v_{\text {orb }}}\right)^{4 / 7},
$$

where $v_{\text {orb }}$ is the internal orbital velocity of the $\mathrm{BBH}$ defined here as the relative velocity between the two BHs assuming a circular orbit. As we only consider the hard binary (HB) limit in this paper $\left(v_{\text {orb }} \gg v\right.$, see e.g. [112]), the above relations imply that $\mathcal{R}_{\mathrm{bs}}$ will always be $<\mathcal{R}_{\mathrm{sS}}$, which leads to the following inequality for the corresponding GW capture cross sections: $\sigma_{\mathrm{bs}}^{<\mathcal{R}} / \sigma_{\mathrm{ss}}^{<\mathcal{R}}<3 \mathcal{N}$.

Finally, as will be shown later in Section III A 3, a highly relevant ratio to consider is $\mathcal{R}_{\mathrm{bs}, \mathrm{ej}} / \mathcal{R}_{\mathrm{ss}}$, where the subscript 'ej' states that $\mathcal{R}_{\mathrm{bs}}$ is here evaluated at the SMA $a=a_{\mathrm{ej}}$, where $a_{\mathrm{ej}}$ is the (maximum) SMA from which the $\mathrm{BBH}$ will get ejected from the cluster through a binary-single interaction [75]. Assuming that each binary-single interaction decreases the SMA of the $\mathrm{BBH}$ by a fixed fraction $\delta$, such that $a \rightarrow \delta a$, then it follows from classical mechanics that $a_{\mathrm{ej}}=(1 / 6)(\phi-1) G m / v_{\mathrm{esc}}^{2}$, where $v_{\text {esc }}$ is the escape velocity of the cluster, and $\phi \equiv 1 / \delta[75]$. By substituting this relation for $a_{\mathrm{ej}}$ into Eq. (9) one finds,

$$
\frac{\mathcal{R}_{\mathrm{bs}, \mathrm{ej}}}{\mathcal{R}_{\mathrm{ss}}}=\left(\frac{\phi-1}{12 f_{\text {ed }}^{2}}\right)^{2 / 7},
$$

where $f_{\text {ed }} \equiv v_{\text {esc }} / v$ (the subscript 'ed' refers to that $f$ is the fraction between the ejection (e) and the dispersion 
(d) velocities, respectively). Assuming $f_{\text {ed }}=5$, and $\delta=$ $7 / 9$ [e.g. 75,112 ], one finds that $\mathcal{R}_{\mathrm{bs}, \mathrm{ej}} / \mathcal{R}_{\mathrm{ss}}=1050^{-2 / 7} \approx$ $7.3^{-1}$. For comparison, if we evaluate this ratio at the HB limit value of the SMA, $a_{\mathrm{HB}}=(3 / 2) G m / v^{2}$, which is the SMA at which the total energy of the binary, $-G m^{2} /(2 a)$, equals the energy of incoming single encounters, $(1 / 2) \mu v^{2}$, we find $\mathcal{R}_{\mathrm{bs}, \mathrm{HB}} / \mathcal{R}_{\mathrm{ss}} \approx 1$. Note that this also follows directly from Eq. (9) when $v \approx v_{\text {orb }}$. For our chosen values it therefore follows that $8 \lesssim \sigma_{\mathrm{bs}}^{<\mathcal{R}} / \sigma_{\mathrm{ss}}^{<\mathcal{R}} \lesssim$ 60 .

\section{RATES}

In this section we convert the cross sections derived in the above Section II to formation rates. We assume steady state in all our calculations, which of course is a simplification of how real clusters evolve; however, this assumption allows us to explore the rates and observables in closed form expressions, which provides crucial guidance to what to focus on and include in more sophisticated numerical simulations. Also, this allows for an easier comparison to the recent analytical literature on both binary-single and single-single GW mergers forming in both GN and GCs. We proceed below by first deriving a few general relations, after which we apply these to study the binary-single and single-single rates of GW capture mergers from two different cluster models; a uniform density model and the Plummer's sphere model. Corresponding observables, such as the GW peak frequency and $\mathrm{BBH}$ orbital eccentricity will be discussed in Section IV.

\section{A. General Relations}

In the following we present general relations for deriving absolute and relative rates of GW mergers resulting from single-single and binary-single interactions, respectively.

\section{Single-Single Rates}

We consider a spherical cluster with mass density profile $\rho(r)$ and corresponding number density profile $n(r)=\rho(r) / m$, consisting of identical compact objects with mass $m$. In a radial shell with width $d r$ at radial position $r$, the number of single-single encounters with $r_{\mathrm{p}}<R_{\mathrm{sS}}(r)$ per time interval $d t$ is given by,

$$
d \Gamma_{\mathrm{ss}}(r)=n(r) \sigma_{\mathrm{ss}}^{<R}(r) v(r) \times n(r) 4 \pi r^{2} d r \times \frac{1}{2},
$$

where the first term is the rate of encounters with $r_{\mathrm{p}}<$ $R_{\mathrm{ss}}(r)$ a single object experiences, the second term is the number of single objects within the considered shell, and the third term corrects for that the singles are both targets and encounters (two singles result in one encounter). From here we do not explicitly show if a quantity $x$ is dependent on, e.g., $r$ by writing $x(r)$. This is done to limit the length of our expressions. Using the above relation from Eq. (11), the total single-single rate of encounters with $r_{\mathrm{p}}<R_{\mathrm{ss}}$ from the entire cluster is now given by,

$$
\Gamma_{\mathrm{ss}}=\frac{8 \pi^{2} G}{m} \int_{0}^{\infty} \frac{R_{\mathrm{ss}} \rho^{2}}{v} r^{2} d r .
$$

Note here that we have allowed for the characteristic encounter distance $R_{\mathrm{ss}}$ to be a function of $r$. A variable $R_{\mathrm{ss}}$ will be important to include when deriving the total rate of single-single GW capture mergers. The above equation can also be written in the following form,

$$
\begin{aligned}
\Gamma_{\mathrm{ss}} & =n_{0} \sigma_{\mathrm{ss}, 0}^{<R} v_{0} \frac{1}{2} N_{\mathrm{s}} \times \frac{4}{3} \frac{\pi r_{\mathrm{s}}^{3} \rho_{0}}{M} \int_{0}^{\infty} \frac{\tilde{R}_{\mathrm{ss}} \tilde{\rho}^{2}}{\tilde{v}} 3 \tilde{r}^{2} d \tilde{r} \\
& =n_{0} \sigma_{\mathrm{ss}, 0}^{<R} v_{0} \frac{1}{2} N_{\mathrm{s}} \times \xi_{s s},
\end{aligned}
$$

where $N_{\mathrm{s}}$ is the total number of singles, $M=N_{\mathrm{s}} \times m$ is the total mass, $\tilde{R}_{\mathrm{ss}}=R_{\mathrm{ss}} / R_{\mathrm{ss}, 0}, \tilde{\rho}=\rho / \rho_{0}, \tilde{v}=v / v_{0}$, $\tilde{r}=r / r_{\mathrm{s}}$, and

$$
\xi_{s s} \equiv \frac{4}{3} \frac{\pi r_{\mathrm{s}}^{3} \rho_{0}}{M} \int_{0}^{\infty} \frac{\tilde{R}_{\mathrm{ss}} \tilde{\rho}^{2}}{\tilde{v}} 3 \tilde{r}^{2} d \tilde{r} .
$$

Here the subscript ' 0 ' denotes the corresponding quantity has to be evaluated at $r=0$ (central cluster values), and $r_{\mathrm{s}}$ is a characteristic scale. It is convenient to consider this notation of the rate, as $\xi_{s s}=1$ for a simple uniform sphere with constant $R_{\mathrm{ss}}$. In other words, the last term, $\xi_{s s}$, represents essentially an 'efficiency factor' that depends on how the $N_{\mathrm{s}}$ objects distribute within the cluster.

\section{Binary-Single Rates}

We now turn to a derivation of the rate of close twobody encounters forming during binary-single interactions. To analytically calculate this we here make use of the simple dynamical model described in $[75,90]$. In short, we assume that all binaries form with a SMA equal to their HB value, $a_{\mathrm{HB}}$ (see Section II C), after which a given binary undergoes interactions with incoming singles that each decreases the binary SMA from $a \rightarrow \delta a$. This in-cluster hardening process continues until the binary SMA falls below the critical ejection value $a_{\mathrm{ej}}$ (see Section IIC), at which the binding energy released in a single binary-single interaction leads to ejection of the binary from its cluster. The average rate of encounters with $r_{\mathrm{p}}<R_{\mathrm{bs}}$ forming during binary-single interactions can in this model be approximated by $\Gamma_{\mathrm{bs}} \approx N_{\mathrm{bs}} / T_{\mathrm{ej}}$, where $N_{\mathrm{bs}}$ is the total number of encounters with $r_{\mathrm{p}}<R_{\mathrm{bs}}$ forming during the hardening binary-single interactions of the bi- 
nary from $a_{\mathrm{HB}}$ to $a_{\mathrm{ej}}$, and $T_{\mathrm{ej}}$ is the time it takes for the binary to transition from $a_{\mathrm{HB}}$ to $a_{\mathrm{ej}}$.

For estimating this rate, we start by deriving $N_{\mathrm{bs}}$. We do this by first considering the differential version of Eq. $(5), d N_{\mathrm{bs}}=\left(2 R_{\mathrm{bs}} / a\right) \mathcal{N} d k$, where $k$ here refers to hardening step $k$, i.e. $a(k)=a_{\mathrm{HB}} \delta^{k}$. By changing variable from $k$ to SMA $a$ using the relation $d a=-a(1-\delta) d k$ now follows,

$$
N_{\mathrm{bs}}=\frac{2 \mathcal{N}}{1-\delta} \int_{a_{\mathrm{ej}}}^{a_{\mathrm{HB}}} \frac{R_{\mathrm{bs}}}{a^{2}} d a,
$$

where we here, and in the rest of the paper, assume that the probability for a $\mathrm{BBH}$ to merge inside the cluster is $<1$, which is a reasonable assumption for standard GCs. As a cross check, if we here substitute $R_{\mathrm{bs}}$ with the GW inspiral merger distance $\mathcal{R}_{\text {bs }}$ given by Eq. (7), then one finds that the number of such mergers evaluates to $N_{\mathrm{bs}}^{<\mathcal{R}} \approx 7 P_{\mathrm{bs}, \mathrm{ej}}^{<\mathcal{R}} /(5(1-\delta))$, where $P_{\mathrm{bs}, \mathrm{ej}}^{<\mathcal{R}}$ is given by Eq. (5) evaluated at $a=a_{\mathrm{ej}}$. This relation was found in [75], which serves as an excellent confirmation of our relations so far. Note here that the 'enhancement factor' from including the whole binary-single hardening sequence, and not only the final SMA $\left(a=a_{\mathrm{ej}}\right)$, evaluates to $7 /(5(1-\delta))=63 / 10$.

We now turn to deriving the time interval $T_{\text {ej }}$, for which we assume the binaries distribute near the cluster center, such that they only relate to the cluster properties through the central values, $n_{0}, v_{0}$. In this approximation we continue by first use that the time between binarysingle interactions is given by the inverse binary-single encounter rate, $\left(n_{0} \sigma_{\mathrm{bs}} v_{0}\right)^{-1}$, which can be converted to the differential form $d t=\left(n_{0} \sigma_{\mathrm{bs}} v_{0}\right)^{-1} d k$, where $t$ here denotes time. Using $d a=-a(1-\delta) d k$, we can now write the total time it takes for a given binary to transition from $a_{\mathrm{HB}}$ to $a_{\mathrm{ej}}$ through binary-single scatterings in the following way,

$$
\begin{aligned}
T_{\mathrm{ej}} & =\int_{a_{\mathrm{ej}}}^{a_{\mathrm{HB}}} \frac{1}{n_{0} \sigma_{\mathrm{bs}} v_{0}} \frac{d a}{a(1-\delta)}, \\
& \approx \frac{(6 \pi G)^{-1}}{(1-\delta)} \frac{v_{0}}{n_{0}} \frac{m^{-1}}{a_{\mathrm{ej}}},
\end{aligned}
$$

where we have used Eq. (4), and for the last term assumed that $a_{\mathrm{HB}} \gg a_{\mathrm{ej}}$. We note here that this is also approximately the inverse rate of binary ejections, i.e., $\Gamma_{\mathrm{ej}} \approx 1 / T_{\mathrm{ej}}$.

By the use of Eq. (15) and Eq. (16), and that $\Gamma_{\mathrm{bs}} \approx$ $N_{\mathrm{bs}} / T_{\mathrm{ej}}$, we now finally find,

$$
\begin{aligned}
\Gamma_{\mathrm{bs}} & =n_{0} \sigma_{\mathrm{bs}, \mathrm{ej}}^{<R} v_{0} N_{\mathrm{b}} \times \int_{1}^{a_{\mathrm{HB}}^{\prime}} \frac{R_{\mathrm{bs}}^{\prime}}{{a^{\prime}}^{2}} d a^{\prime} \\
& =n_{0} \sigma_{\mathrm{bs}, \mathrm{ej}}^{<R} v_{0} N_{\mathrm{b}} \times \xi_{\mathrm{bs}},
\end{aligned}
$$

where $N_{\mathrm{b}}$ is the number of binaries, $a^{\prime}=a / a_{\mathrm{ej}}, R_{\mathrm{bs}}^{\prime}=$ $R_{\mathrm{bs}} / R_{\mathrm{bs}, \mathrm{ej}}, \sigma_{\mathrm{bs}, \mathrm{ej}}^{<R}$ denotes the cross section evaluated at $a=a_{\mathrm{ej}}$, and

$$
\xi_{b s} \equiv \int_{1}^{a_{\mathrm{HB}}^{\prime}} \frac{R_{\mathrm{bs}}^{\prime}}{a^{\prime 2}} d a^{\prime} .
$$

As seen, for a constant value of $R_{\mathrm{bs}}^{\prime}$ the scale-factor $\xi_{b s} \approx 1$ for $a_{\mathrm{HB}}^{\prime} \gg 1$. The factor $\xi_{b s}$ therefore represents the integrated effect from including a scale dependent $R_{\mathrm{bs}}$, as e.g. the GW capture distance $\mathcal{R}_{\mathrm{bs}}$ given by Eq. (7). As described in [73], the characteristic distance for dissipative captures, including tidal and GW emission captures, can often be approximated by $R \propto a^{\beta}$, where $\beta$ relates to the energy loss in question, $\Delta E$, as $\Delta E \propto r_{\mathrm{p}}^{-\beta}$. As seen, for this class of ' $\beta$-models' the efficiency factor is given by $\xi_{b s}=(1-\beta)^{-1}$ for $a_{\mathrm{HB}}^{\prime} \gg 1$.

\section{Binary-Single vs. Single-Single}

Having derived general forms for the close encounter rate from both single-single, $\Gamma_{\mathrm{ss}}$ (Section III A 2), and binary-single, $\Gamma_{\mathrm{bs}}$ (Section III A 2), we are now in a position to compare the two. Using Eq. (17) and Eq. (13) we find,

$$
\frac{\Gamma_{\mathrm{bs}}}{\Gamma_{\mathrm{ss}}} \approx 2 \frac{N_{\mathrm{b}}}{N_{\mathrm{s}}} \frac{\sigma_{\mathrm{bs}, \mathrm{ej}}^{<R}}{\sigma_{\mathrm{ss}, 0}^{<R}} \frac{\xi_{\mathrm{bs}}}{\xi_{\mathrm{ss}}}=6 F_{\mathrm{bs}} \mathcal{N} \times \frac{R_{\mathrm{bs}, \mathrm{ej}}}{R_{\mathrm{ss}, 0}} \frac{\xi_{\mathrm{bs}}}{\xi_{\mathrm{ss}}},
$$

where the factor $F_{\mathrm{bs}}$ denotes the binary fraction, $F_{\mathrm{bs}}=$ $N_{\mathrm{b}} / N_{\mathrm{s}}$. This simple expression constitutes one of our main results from this paper. In the following sections we will evaluate this ratio for different characteristic distances (constant $R$ and GW capture $\mathcal{R}$ ) and cluster profiles ('MODEL I' and 'MODEL II'). The main purpose of this is to illustrate that single-single interactions could contribute with a non-negligible and unique population of $\mathrm{BBH}$ mergers.

\section{B. MODEL I: Uniform Density Sphere}

We here consider a BH subsystem described by a uniform density $\rho$ and velocity dispersion $v$. This example therefore represents a classical ' $n \sigma v$ ' estimate with a finite number of objects, $N_{\mathrm{s}}$. Considering first the singlesingle population, it is here natural to set $r_{\mathrm{s}}$ equal to the size of the cluster, which implies that $\tilde{\rho}=1$ and $\tilde{v}=1$ for $r<r_{\mathrm{s}}$, and that $\tilde{R}_{\mathrm{ss}}=1$ and $\xi_{s s}=1$ for a constant $R_{\mathrm{ss}}$ and the GW capture $\mathcal{R}_{\mathrm{ss}}$. For binary-single interactions, the efficiency factor $\xi_{b s}$ from Eq. (18) will in contrast only $=1$ for constant $R_{\mathrm{bs}}$ encounters, but not for GW captures with varying $\mathcal{R}_{\text {bs }}$. Below we study the rate of close encounters and GW capture mergers for this model. 


\section{Close Encounters}

We start by writing out the rate of single-single encounters with $r_{\mathrm{p}}<R_{\mathrm{ss}}$, where $R_{\mathrm{ss}}$ is assumed constant. Since $\xi_{\mathrm{ss}}=1$, as argued above, then the rate is given by,

$$
\Gamma_{\mathrm{ss}} \approx n \sigma_{\mathrm{ss}}^{<R} v \frac{1}{2} N_{\mathrm{s}}
$$

which follows from Eq. (13). This is not surprisingly the usual rate of encounters per single, ' $n \sigma v$ ', weighted by the total number of singles divided by two, $N_{\mathrm{s}} / 2$ (see also [114]).

We now consider the rate of binary-single encounters with $r_{\mathrm{p}}<R_{\mathrm{bs}}$, where also $R_{\mathrm{bs}}$ is here assumed constant. Since $\xi_{\mathrm{bs}}=1$ also in this case, the rate is simply given by,

$$
\Gamma_{\mathrm{bs}} \approx n \sigma_{\mathrm{bs}}^{<R} v N_{\mathrm{b}}
$$

where we have used Eq. (17). Remember here that the cross section $\sigma_{\mathrm{bs}}^{<R}$ is $\propto m R_{\mathrm{bs}} / v^{2}$, and therefore independent of the SMA $a$.

If we now consider the special case for which $R_{\mathrm{ss}}=R_{\mathrm{bs}}$, then the ratio between the two rates, $\Gamma_{\mathrm{bs}}$ and $\Gamma_{\mathrm{ss}}$, reduces to the following expression

$$
\frac{\Gamma_{\mathrm{bs}}}{\Gamma_{\mathrm{ss}}} \approx 6 F_{\mathrm{bs}} \mathcal{N},
$$

where we have used Eq. (19). This explicitly illustrates that $\Gamma_{\mathrm{bs}} / \Gamma_{\mathrm{ss}}$ is independent of the cluster density, its velocity dispersion, and the orbital evolution of the interacting binaries. Instead, it essentially only depends on the binary fraction $F_{\mathrm{bs}}$. As seen, the two rates are therefore comparable when the binary fraction $F_{\mathrm{bs}} \approx(6 \mathcal{N})^{-1}$, from which we conclude that for this model $\Gamma_{\mathrm{ss}} \approx \Gamma_{\mathrm{bs}}$ when $F_{\mathrm{bs}} \approx 1 / 120 \sim 1 \%$. Therefore, for our considered model, single-single and binary-single encounters with $r_{\mathrm{p}}<R_{\mathrm{ss}}=R_{\mathrm{bs}}$ contribute at the same level when the binary fraction is at the percent level. Interestingly, this is exactly the level that is observed in state-of-the-art GC simulations, which explains why single-single interactions might actually contribute with a non-negligible fraction of BBH mergers. An illustration of this is shown in Fig. 2 .

As will be discussed later, a constant $R$, as considered here, is important for understanding the rate of GW sources with a particular GW peak frequency (the GW frequency where most of the power is outputted) which to leading order only depends on the $\mathrm{BBH}$ peri-center distance $r_{\mathrm{p}}$ (see Eq. (35)). Below we continue by deriving the rate of $\mathrm{GW}$ mergers forming within the scale dependent capture distance $\mathcal{R}$.

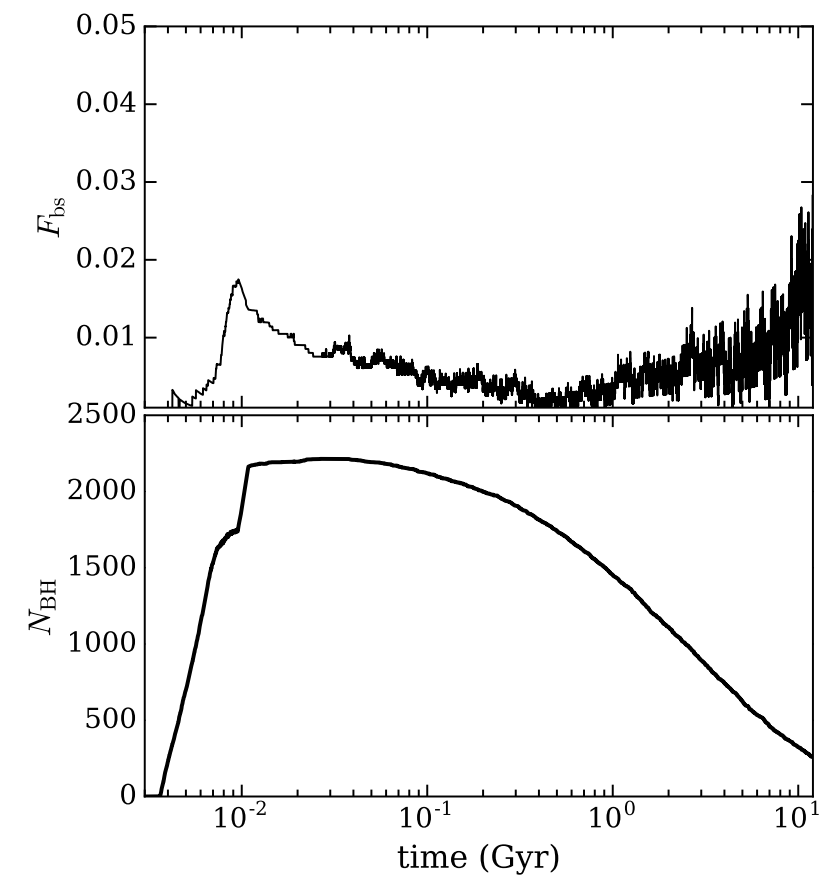

FIG. 2. Evolution of the $\mathrm{BH}$ population in a representative GC (initial conditions: 10 $0^{6}$ stars, $5 \%$ binary fraction, and virial radius $=1 \mathrm{pc}$ ), simulated using the CMC code [78] that is based on a MC Henon solver. Top: Number of BBHs relative to the total number of single BHs, $F_{\mathrm{bs}}=N_{\mathrm{b}} / N_{\mathrm{s}}$, as a function of time after the formation of the GC. Bottom: Total number of single $\mathrm{BHs}$ in the $\mathrm{GC}$ as a function of time. The initial rise is due to single and binary star evolution, where the later decline arises from dynamical interactions that both eject single BHs from the cluster, and swap them into binaries that eventually merge. As seen, the binary fraction stays at the percent level throughout the entire life of the GC, which implies that the rate of single-single GW capture mergers are likely to be similar to the rate of mergers forming during binary-single interactions (see e.g. Eq. (22) and Eq. (24)). However, neither the binary fraction nor the total number of single BHs stays exactly constant, and one therefore expects that the relative rate of single-single GW captures to change as a function of time. The associated time dependent change of the distribution of GW peak frequency and orbital eccentricity will make it possible for next generation GW observatories to better constrain the dynamical processes that drive BBHs to merger in dense stellar systems [e.g. 115].

\section{GW Mergers}

We now derive the rates of single-single and binarysingle mediated GW mergers for our considered uniform cluster model. One expects a different ratio than the one derived in Eq. (22), as $R$ is now no longer constant for the binary-single interactions.

We start by deriving the single-single GW capture rate. In this case, the characteristic distance $R_{\mathrm{ss}}=\mathcal{R}_{\mathrm{ss}}$, but since the cluster is assumed uniform then $\widetilde{R}_{\mathrm{ss}}$ is still $=1$. The single-single GW capture rate is therefore simply 
given by

$$
\Gamma_{\mathrm{ss}} \approx n \sigma_{\mathrm{ss}}^{<\mathcal{R}} v \frac{1}{2} N_{\mathrm{s}}
$$

where $\sigma_{\mathrm{ss}}^{<\mathcal{R}}$ is evaluated using Eq. (1), and Eq. (2).

We now derive the rate of GW inspiral mergers forming during binary-single interactions. From using Eq. (7) it follows that $R_{\mathrm{bs}}^{\prime}=\left(a / a_{\mathrm{ej}}\right)^{2 / 7}=a^{\prime 2 / 7}$, from which we now find using Eq. (18) that,

$$
\xi_{b s}=\int_{1}^{a_{\mathrm{HB}}^{\prime}} \frac{a^{\prime 2 / 7}}{a^{\prime 2}} d a^{\prime} \approx \frac{7}{5} .
$$

For this we assumed the limit $a_{\mathrm{HB}} \gg 1$. Note here that this result also follows from setting $\beta=7 / 2$ in the general relation $\xi_{b s}=(1-\beta)^{-1}$ derived below Eq. (18). With this value of $\xi_{b s}$, the rate of GW inspiral mergers forming during binary-single interactions is given by,

$$
\Gamma_{\mathrm{bs}}=\frac{7}{5} n \sigma_{\mathrm{bs}, \mathrm{ej}}^{<\mathcal{R}} v N_{\mathrm{b}} .
$$

The scale dependence of $\mathcal{R}_{\mathrm{ss}}$ gives rise to a small increase of $7 / 5$, compared to just evaluating the rate at the ejection limit $a=a_{\mathrm{ej}}$.

Comparing the rates of GW mergers from the binarysingle and single-single channels we find,

$$
\frac{\Gamma_{\mathrm{bs}}}{\Gamma_{\mathrm{ss}}} \approx 6 F_{\mathrm{bs}} \mathcal{N} \times\left(\frac{\phi-1}{12 f_{\mathrm{ed}}^{2}}\right)^{2 / 7} \frac{7}{5},
$$

where we have used Eq. (19) and Eq. (10). As seen, when accounting for all the GW mergers that form through single-single and binary-single interactions, and not only those for which $r_{\mathrm{p}}<R_{\mathrm{ss}}=R_{\mathrm{bs}}$ as we did in the above Section III B 1, the relative GW merger rate from singlesingle interactions increases by a factor of $(5 / 7) 1050^{2 / 7} \approx$ 5.2 for $f_{\text {ed }}=5$ and $\delta=7 / 9$. Therefore, in this case $\Gamma_{\mathrm{ss}} \approx \Gamma_{\mathrm{bs}}$ for $F_{\mathrm{bs}} \approx 5.2 / 120 \sim 5 \%$. This indicates that single-single GW captures very well could play a role in the formation of eccentric in-cluster mergers, as $F_{\mathrm{bs}}$ is likely $<5 \%$ for standard GCs, as shown by Fig. 2. We proceed below by exploring how our results from this section change when considering a more realistic density profile for the single $\mathrm{BH}$ population.

\section{MODEL II: Plummer's Sphere}

Real clusters are not described by the simple uniform density sphere that we considered in the above Section III B. Instead, relaxation processes generally drive systems to a state described by a profile having a high density in the center and a low in the outskirts [e.g. 116]. To study the effect of a more realistic profile, we here derive absolute and relative rates assuming the single $\mathrm{BHs}$ distribute according to the well-known Plummer's sphere [117]. We choose this as it allows for a full analytical treatment of the problem in contrast to other families of profiles.

To start, we first introduce the mass density profile of the Plummer's sphere, which is given by,

$$
\rho=\frac{3 M}{4 \pi b^{3}}\left(1+\frac{r^{2}}{b^{2}}\right)^{-5 / 2},
$$

and the corresponding velocity dispersion,

$$
v^{2}=\frac{1}{6} \frac{G M}{b}\left(1+\frac{r^{2}}{b^{2}}\right)^{-1 / 2},
$$

where $M$ is the total cluster mass, and $b$ is a characteristic scale [117]. Inserting these two expressions into Eq. (14), one finds that the single-single efficiency factor for this profile reduces to the following form

$$
\xi_{s s}=3 \int_{0}^{\infty} \tilde{R}_{\mathrm{ss}}\left(1+x^{2}\right)^{-19 / 4} x^{2} d x .
$$

The binary-single efficiency factor $\xi_{b s}$ is unchanged from what was found in the above Section III B, as we assume the binary-single encounter rate only depends on the central properties of the cluster. Finally, from using that the potential of the Plummer's sphere is given by $\phi(r)=$ $-G M /\left(b \sqrt{1+(r / b)^{2}}\right)$ and that $v_{\text {esc }}(r)=\sqrt{-2 \phi(r)}$ it directly follows that $v_{\text {esc }, 0} / v_{0}=\sqrt{12} \approx 3.5$. As this is very close to our 'fiducial' chosen value of $f_{\text {ed }}$, we will still be using $f_{\text {ed }}=5$ in the following sections to make comparisons more clear. Below we consider the absolute and relative rate of close encounters and GW mergers for the Plummer's sphere.

\section{Close Encounters}

We start by considering the rate of single-single encounters with $r_{\mathrm{p}}<R_{\mathrm{ss}}$, where $R_{\mathrm{ss}}$ is assumed constant. In this case $\tilde{R}_{\mathrm{ss}}=1$, from which we find by the use of Eq. (29) that

$$
\xi_{s s}=3 \int_{0}^{\infty}\left(1+x^{2}\right)^{-19 / 4} x^{2} d x=\frac{3 \sqrt{\pi}}{4} \frac{\mathscr{G}(13 / 4)}{\mathscr{G}(19 / 4)},
$$

where $\mathscr{G}(z)=\int_{0}^{\infty} x^{z-1} e^{-x} d x$ denotes the well-known Gamma function. With this factor it is now straight forward to derive the single-single rate using Eq. (13). The question is now, does the change from a uniform sphere to a more realistic density profile leads to an increase or a decreases of the single-single close encounter rate? As seen here, for the Plummer's sphere $\xi_{s s}=$ $(3 \sqrt{\pi} / 4) \mathscr{G}(13 / 4) / \mathscr{G}(19 / 4) \approx 0.2$, which means that the rate from distributing $N_{\mathrm{s}}$ singles in a Plummer's sphere is about 5 times smaller than if one distributes them in a uniform sphere. The resulting ratio between the binary-single and single-single close encounter rates for 
$R_{\mathrm{ss}}=R_{\mathrm{bs}}$ is given by,

$$
\frac{\Gamma_{\mathrm{bs}}}{\Gamma_{\mathrm{ss}}} \approx 6 F_{\mathrm{bs}} \mathcal{N} \times \frac{4}{3 \sqrt{\pi}} \frac{\mathscr{G}(19 / 4)}{\mathscr{G}(13 / 4)},
$$

where we have used Eq. (19), and the above Eq. (30). From this we conclude that $\Gamma_{\mathrm{ss}} \approx \Gamma_{\mathrm{bs}}$ for $F_{\mathrm{bs}} \approx 1 / 600 \sim$ $0.15 \%$, which suggests that $\mathrm{BH}$ subsystems with a realistic density profile is unlikely to have single-single captures significantly contributing to encounters with $r_{\mathrm{p}}<R_{\mathrm{ss}}=R_{\mathrm{bs}}$. Below we study the absolute and relative rate of GW mergers.

\section{2. $G W$ Mergers}

For single-single $\mathrm{GW}$ capture mergers $R_{\mathrm{ss}}=\mathcal{R}_{\mathrm{ss}}$, which implies that $\tilde{R}_{\mathrm{SS}}=\left(v / v_{0}\right)^{-4 / 7}$, where we have used Eq. (2). Plugging this expression into Eq. (29), and by the use of Eq. (28), we find the following value for the single-single efficiency factor,

$$
\xi_{s s}=3 \int_{0}^{\infty}\left(1+x^{2}\right)^{-129 / 28} x^{2} d x=\frac{3 \sqrt{\pi}}{4} \frac{\mathscr{G}(87 / 28)}{\mathscr{G}(129 / 28)},
$$

which (also) evaluates to $\approx 0.2$. Therefore, the rate of single-single GW capture mergers is also greatly reduced for the Plummer's sphere, compared to the uniform model. Finally, we can also here compare to the rate from binary-single GW mergers. By the use of Eq. (19), the relation shown in Eq. (10), and our derived values for $\xi_{s s}$ (Eq. (32)) and $\xi_{b s}$ (Eq. (24)), we find

$$
\frac{\Gamma_{\mathrm{bs}}}{\Gamma_{\mathrm{ss}}} \approx 6 F_{\mathrm{bs}} \mathcal{N} \times\left(\frac{\phi-1}{12 f_{\mathrm{ed}, 0}^{2}}\right)^{2 / 7} \frac{7}{5} \frac{4}{3 \sqrt{\pi}} \frac{\mathscr{G}(129 / 28)}{\mathscr{G}(87 / 28)},
$$

where $f_{\mathrm{ed}, 0} \equiv v_{\mathrm{esc}, 0} / v_{0}$. For $\delta=7 / 9$ and $f_{\mathrm{ed}, 0}=5$, the term after the ' $X$ '-sign evaluates to $\approx 1$. We therefore conclude that even when accounting for all GW mergers that can form through single-single and binary-single interactions, the two rates are only expected to be similar for $F_{\mathrm{bs}} \approx 1 / 120 \sim 1 \%$. However, as previously described, and also shown in Fig. 2, $F_{\mathrm{bs}}$ is in fact likely to be at the percent level (see Fig. 2).

\section{OBSERVABLE IMPLICATIONS}

Having shown using analytical arguments that $\mathrm{BH}$ single-single GW captures in GCs might give rise to an observable population of mergers, we now explore what the observable characteristics are of these mergers. In particular, we explore if single-single GW capture mergers can be distinguished from the other $\mathrm{BBH}$ merger types in GCs. These include BBHs dynamically ejected from their host cluster, BBHs merging in-between their binary-single interactions, and BBHs merging during their binary-single interactions. In the following sec-

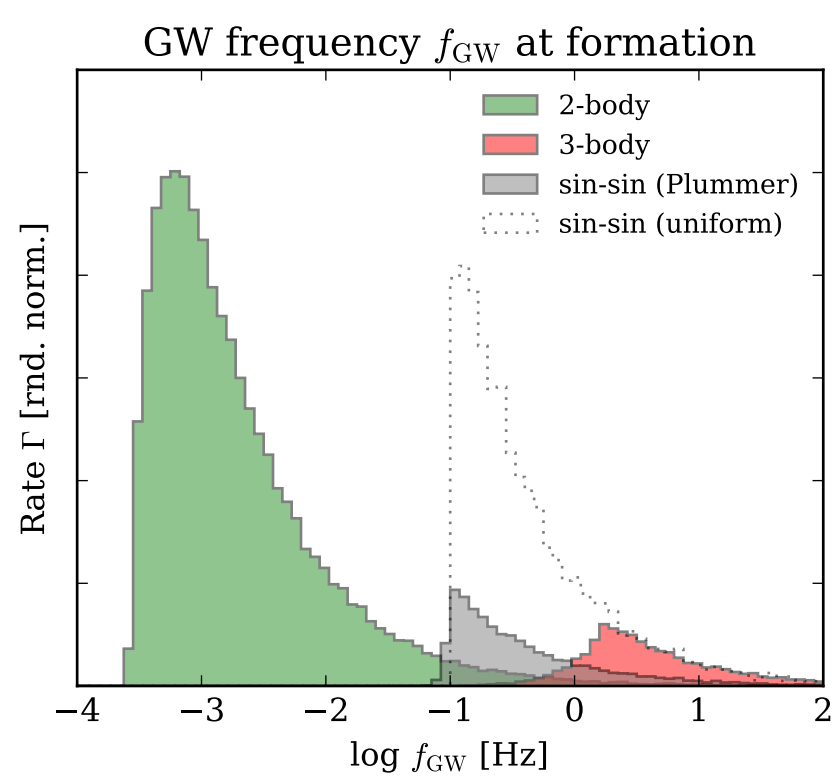

FIG. 3. Distribution of GW peak frequency $\left(f_{\mathrm{GW}}\right)$ of the $\mathrm{BBH}$ mergers forming in our cluster model described in Section IV A. Each $f_{\mathrm{GW}}$ is derived at the time of formation of the $\mathrm{BBH}$ in question, i.e. right when our numerical routine identifies it as a BBH that will inspiral and merge. Since $f_{\mathrm{GW}}$ to leading order only depends on the $\mathrm{BBH}$ peri-center distance, a $\mathrm{BBH}$ with a given $f_{\mathrm{GW}}$ will undergo most of its inspiral at that $f_{\mathrm{GW}}$. When a $\mathrm{BBH}$ has circularized it will move towards the right in the above figure, and thereby enter higher frequencies with a lower eccentricity than its initial value. The '2-body' (green) and '3-body' (red) distributions show results for BBHs merging in-between and during their binary-single interactions, respectively. The 'sin-sin (Plummer)' and the 'sin-sin (uniform)' distributions show results from single-single GW captures forming in a Plummer's sphere and a sphere with a uniform distribution of single BHs, respectively. As seen, assuming a uniform distribution predicts a surprisingly high fraction of single-single GW capture mergers. Assuming a more realistic density profile, such as the Plummer's sphere, results in a significant reduction of the rate. Results are discussed in section IV B 1, and corresponding eccentricity distributions at $1 \mathrm{~Hz}$ and $10 \mathrm{~Hz}$ are shown in Fig. 4.

tions we will refer to these GW merger types as ejected mergers, 2-body mergers, and 3-body mergers, respectively, to shorten labels and descriptions (see also [68]). As pointed out by [e.g. 64, 68, 78, 105], these BBH merger types have different eccentricity- and GW peak frequency distributions. For example, in [64] it was pointed out that 2-body mergers will naturally form near the LISA band with high eccentricity, where 3-body mergers will form at higher frequencies making them eccentric LIGO sources [70].

We here explore how the single-single GW capture mergers will distribute, and in particular what their properties are at $1 \mathrm{~Hz}$ and $10 \mathrm{~Hz}$ in $\mathrm{GW}$ peak frequency. The $1 \mathrm{~Hz}$ regime is potentially interesting for the planned 
deci-Hertz observatories DECIGO and Tian Qin, where the $10 \mathrm{~Hz}$ limit is naturally interesting for currently operating observatories such as LIGO/VIRGO, but has also relevance for third generation observatories including ET/CE, which likely will be able to resolve eccentricities down to $\sim 0.01$ in this range [118]. It is clear that single-single GW captures are not the dominating source of BBH mergers forming in GCs; however, with future observatories such as $\mathrm{ET} / \mathrm{CE}$ we are entering an age where we can expect to see every $\mathrm{BBH}$ merger in the visible Universe. Therefore, it is important to have a solid understanding for how BBH mergers might form in different environments, and what their corresponding observable distributions can tell us about their host systems. Below we present results from a simple analytical MC method.

\section{A. Analytical Monte Carlo Approach}

To derive BBH merger distributions with the inclusion of single-single interactions, we start by deriving the distribution of 2-body and 3-body mergers using the semianalytical MC approach first described in [64]. After this, we super impose the distribution from single-single interactions given some density profile. As for our analytical results, the 2-body and 3-body mergers are all derived assuming that the binary-single encounter rate is determined by the central quantities of the cluster only. In the following we describe this procedure in detail.

\section{Modeling Binary-Single Mergers}

For building up the distribution of 2-body and 3-body mergers we follow a large ensemble of uncorrelated BBHs undergoing binary-single interactions in an environment described by a constant velocity dispersion, $v$, and number density, $n$, equal to the central values of the cluster. Following [64], each of these BBHs are assumed to form with an initial SMA equal to their hard-binary value $a_{\mathrm{HB}}$; however, for GCs the exact upper value of the SMA $a$ is not important as long as $a_{\mathrm{HB}} \gg a_{\mathrm{ej}}$. Considering now the evolution of one of these BBHs, this $\mathrm{BBH}$ will after formation undergo strong binary-single interactions, each of which is assumed to lead to a constant decrease in the SMA from $a$ to $\delta a$. At the same time, the interactions will also change the eccentricity of the $\mathrm{BBH}$, where we here assume this change is sampled from the distribution $P(e)=2 e$ [112]. In the point-particle Newtonian limit, this series of binary-single interactions will always end with a dynamical ejection of the $\mathrm{BBH}$ from its host cluster when its SMA falls below $a_{\mathrm{ej}}$. This classical hardening process therefore leads to an ejection of BBHs with a SMA $\sim a_{\mathrm{ej}}$, and an eccentricity distribution that is thermally distributed; however, when GR effects are included in this process, a given BBH can also merge inside its cluster through (at least) 2-body and 3- body mergers. To account for these two in-cluster merger types we follow the approach outlined in the paragraphs below.

3-body Mergers: For determining if a BBH merges during a strong 3-body interaction, we model this often highly chaotic and resonating state using the approach put forward in [75] and briefly described in Section II B. In short, the interaction is here divided up into $\mathcal{N}$ IMSs, each of which is described by a BBH with a bound single $\mathrm{BH}$. For each IMS we assign the corresponding BBH an eccentricity sampled from the distribution $P(e)=2 e$, but keep the SMA fixed to its initial value $a$. To determine if the $\mathrm{BBH}$ undergoes a $\mathrm{GW}$ inspiral merger during this IMS, i.e. merge while the third objects is still bound to it, we compare the energy radiated over one orbit of the $\mathrm{BBH}\left(\Delta E_{\mathrm{GW}}\right)$, to the total orbital energy of the bound 3-body state $\left(E_{\mathrm{bs}}\right)$. If $\Delta E_{\mathrm{GW}}>E_{\mathrm{bs}}$ we label the IMS assembled $\mathrm{BBH}$ as a 3-body merger. This energy threshold is equivalent of saying that the BBH will undergo a GW inspiral merger during the interaction if its pericenter distance $r_{\mathrm{p}}<\mathcal{R}_{\mathrm{bs}}$. If instead $r_{\mathrm{p}}>\mathcal{R}_{\mathrm{bs}}$ the $\mathrm{BBH}$ does not merge during the considered IMS. We repeat this process, i.e. first assigning the IMS assembled $\mathrm{BBH}$ an eccentricity from $P(e)=2 e$ and then compare its $r_{\mathrm{p}}$ to $\mathcal{R}_{\text {bs }}$, up to $\mathcal{N}=20$ times per interaction.

2-body Mergers: If a BBH does not undergo a 3-body merger we determine right after its binary-single interaction if the BBH instead will undergo a 2-body merger. We do this by first assigning an eccentricity to the $\mathrm{BBH}$ sampled from $P(e)=2 e$, after which we calculate its inspiral time $t_{\text {insp }} \propto a^{4}\left(1-e^{2}\right)^{7 / 2}$ and the time it takes for the next strong single encounter to interact with the $\mathrm{BBH}, t_{\text {int }} \propto\left(n_{0} \sigma_{\mathrm{bs}} v_{0}\right)^{-1}$. If $t_{\text {insp }}<t_{\text {int }}$ then we label the $\mathrm{BBH}$ as a 2-body merger, if instead $t_{\text {insp }}>t_{\text {int }}$ the $\mathrm{BBH}$ survives and we move on to the next binary-single interaction.

We repeat this process of checking for 3-body and 2body mergers while the $\mathrm{BBH}$ gradually hardens inside its GC until the BBH either merges or is ejected. For all 2-body and 3-body mergers we record their orbital parameters, $a$ and $e$, at formation, i.e. before they start their inspiral, which allows us to quickly calculate eccentricity and GW peak frequency distributions. These distributions are then normalized by assuming that the $\mathrm{BBHs}$ are formed at a constant rate given by $1 / T_{\mathrm{ej}}$, where $T_{\mathrm{ej}}$ is given by Eq. (16).

This model clearly represents a simplified picture of how a BH population evolves in a real GC; however, it is very fast, have resulted in very precise estimates so far, and provides therefore an ideal test-bed for exploring what effects that might be important to included for an accurate modeling of such systems [e.g. 68].

\section{Modeling Single-Single Mergers}

Calculating the single-single GW capture merger distribution is a well defined problem for a non-evolving den- 

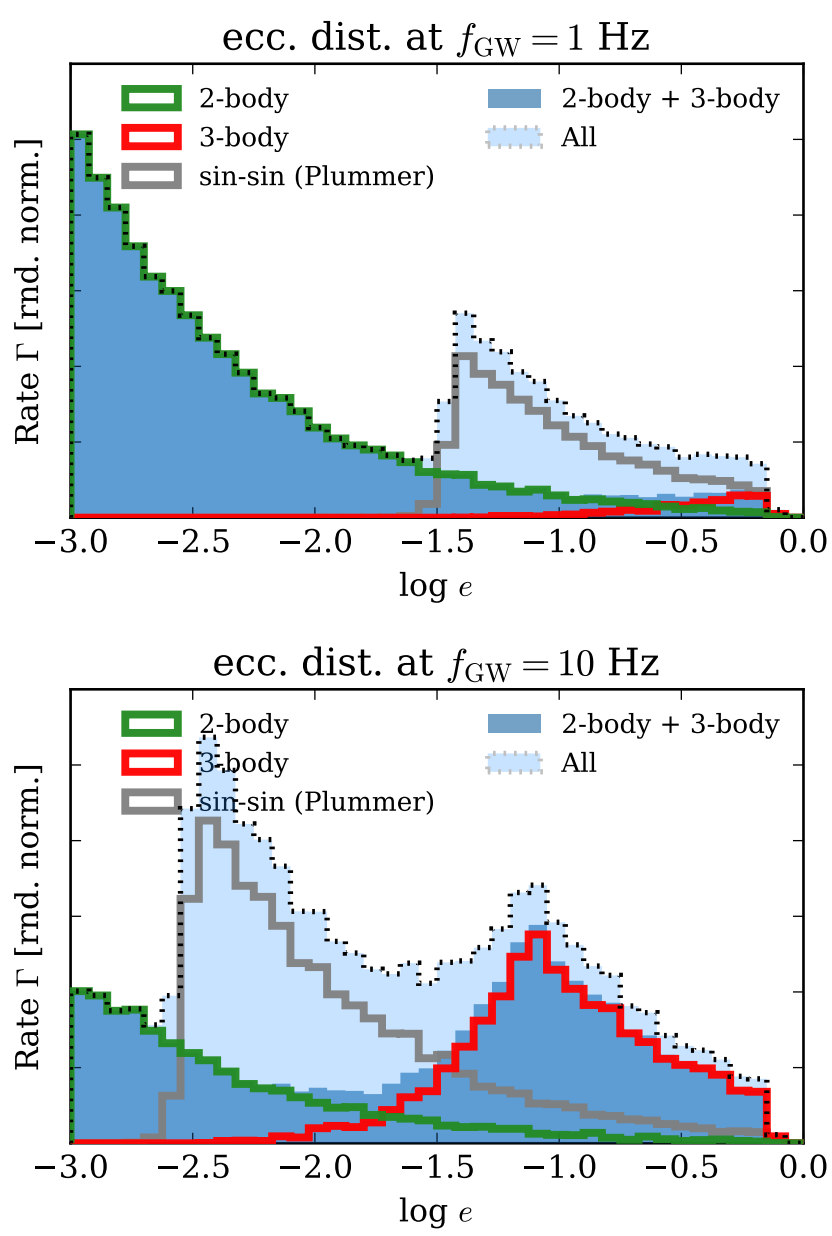

FIG. 4. Orbital eccentricity distributions of the BBH mergers shown in Fig. 3 derived from our model described in Section IV A. The top and the bottom plots show the distributions at $1 \mathrm{~Hz}$ and $10 \mathrm{~Hz}$, respectively. As seen in the top plot, singlesingle GW captures dominate the rate of GW sources with eccentricities $\log (e)>-1.5$ among our considered channels at $1 \mathrm{~Hz}$. This regime is relevant for future planned space borne missions such as DECIGO/Tian Qin. In the bottom plot is seen that single-single GW captures dominate in the regime $-2.5<\log (e)<1.5$, which might be resolvable by future $\mathrm{ET} / \mathrm{CE}$. These results depends on the cluster properties (see e.g. $[37,75])$, which especially implies that future detections can be used to constrain the astrophysical environment of BBH mergers. See Section IV B 2 for further discussions.

sity profile consisting of equal mass objects, and can be done in several ways. For this paper we made a small MC routine that simply samples single-single encounters up to the local GW capture distance $\mathcal{R}_{\mathrm{ss}}$. For each of these encounters we calculate the corresponding orbital parameters, $a=-G m^{2}\left(2 \Delta E_{\mathrm{GW}}+2 E_{\mathrm{ss}}\right)^{-1}$ and $e=1-r_{\mathrm{p}} / a$, of the newly formed $\mathrm{BBH}$ right after capture, from which we can determine eccentricity- and GW peak frequency distributions similar to the binary-single case. In this routine, for the local maximum GW capture distance $\mathcal{R}$ we do not only require that the two single BHs are bound after the encounter, but also that their GW inspiral time, $t_{\text {insp }}(a, e)$, is shorter than the local binary-single interaction time, $t_{\text {int }}(a)$. This requirement results in a local maximum GW capture distance $\mathcal{R}$ that fulfills the following equation,

$$
A m^{9 / 2} \mathcal{R}^{-7 / 2}-\frac{1}{2} \mu v^{2}=B m^{2}\left(\frac{C}{D} \frac{n}{v} \frac{1}{m^{2}}\right)^{2 / 3} \mathcal{R}^{7 / 3},
$$

where $A=(85 \pi / 12) G^{7 / 2} c^{-5}, \quad B=G / 2, \quad C=$ $(768 / 425)\left(2^{7 / 2} 5 / 512\right) G^{-3} c^{5}$, and $D=(6 \pi G)^{-1}$. This unfortunately does not have a closed form solution, but can easily be solved numerically. However, we did not find a significant difference between using the classical capture distance given by Eq. (2), and the one found from the above Eq. (34). Results are discussed in the next section.

\section{B. Results}

In this section we present results from numerical experiments, where we follow a population of binary and single BHs using the routines described in the above Section IV A. All our results are based on a system characterized by $m=20 M_{\odot}, v_{0}=10 \mathrm{kms}^{-1}, n_{0}=10^{5} \mathrm{pc}^{-3}$, $f_{\mathrm{ed}, 0}=5$, and $F_{\mathrm{bs}}=0.01$.

\section{Gravitational Wave Peak Frequency}

We start by considered Fig. 3, which shows the GW peak frequency distributions of the merging population of BBHs right when they form in our MC routine. For making this, we used the orbital parameters $a, e$ of each of the assembled BBHs to derive their corresponding pericenter distance $r_{\mathrm{p}}=a(1-e)$, from which the GW peak frequency, denoted here by $f_{\mathrm{GW}}$, can be approximated by [e.g. 119],

$$
f_{\mathrm{GW}} \approx \frac{1}{\pi} \sqrt{\frac{2 G m}{r_{\mathrm{p}}^{3}}}
$$

Focusing on the single-single (grey/black) and binarysingle (red) mergers we first notice that they peak at different locations: roughly about $10^{-1} \mathrm{~Hz}$ and $10^{0.3} \mathrm{~Hz}$, respectively. For both of these channels, their peak location is near the lowest $f_{\mathrm{GW}}$ at which all of the mergers from the specific channel is able to contribute (see also [67]). The two peak locations can therefore be expressed as, $f_{\mathrm{GW}, \mathrm{ss}}^{\mathrm{peak}} \approx \pi^{-1} \sqrt{2 G m / \mathcal{R}_{\mathrm{ss}, 0}^{3}} \propto v_{0}^{6 / 7} / m$, and $f_{\mathrm{GW}, \mathrm{bs}}^{\text {peak }} \approx \pi^{-1} \sqrt{2 G m / \mathcal{R}_{\mathrm{bs}, \mathrm{ej}}^{3}} \propto v_{\mathrm{esc}}^{6 / 7} / m$, respectively. Now taking the ratio between these two terms we find 
the following relation,

$$
\frac{f_{\mathrm{GW}, \mathrm{bs}}^{\mathrm{peak}}}{f_{\mathrm{GW}, \mathrm{ss}}^{\mathrm{peak}}}=\left(\frac{12 f_{\mathrm{ed}, 0}^{2}}{\phi-1}\right)^{3 / 7}
$$

This interestingly shows that the single-single and binary-single peak locations are separated by a constant factor, that in our model only depends on the properties of the system through $f_{\text {ed, } 0}$. For $\delta=7 / 9$ and $f_{\text {ed, } 0}=5$ follows that $f_{\mathrm{GW}, \mathrm{bs}}^{\text {peak }} / f_{\mathrm{GW}, \mathrm{ss}}^{\text {peak }} \approx 20$, which agrees with the results shown in Fig. 3. As $f_{\text {ed }, 0}$ is always $>1$, our derived ratio further implies that $f_{\mathrm{GW}, \mathrm{bs}}^{\text {peak }} / f_{\mathrm{GW}, \mathrm{ss}}^{\mathrm{peak}}>$ $(12 /(\phi-1))^{3 / 7} \approx 5$, therefore, the peaks will always be separated with $f_{\mathrm{GW}, \mathrm{bs}}^{\text {peak }}>f_{\mathrm{GW}, \mathrm{ss}}^{\text {peak }}$.

If we now consider the actual shapes of the single-single and binary-single distributions in the region where their GW peak frequencies are greater than their corresponding distribution peaks, i.e. for $f_{\mathrm{GW}}>f_{\mathrm{GW}}^{\text {peak }}$, we see that they follow the same functional form. This is most easily seen when comparing the '3-body' with the 'sin-sin (uniform)' distributions. The reason is simply that the single-single and the binary-single cross sections for close encounters with $r_{\mathrm{p}}<R$ are both $\propto R$, as seen in Eq. (1) and Eq. (6). The shape of both of the distributions is therefore given by,

$$
\frac{d \Gamma\left(>f_{\mathrm{GW}}\right)}{d \log f_{\mathrm{GW}}} \propto f_{\mathrm{GW}} \frac{d \sigma(<R)}{d f_{\mathrm{GW}}} \propto f_{\mathrm{GW}}^{-2 / 3},
$$

where we have used that $\sigma^{<R} \propto R$ for both singlesingle and binary-single interactions, and the relation $R \propto f_{\mathrm{GW}}^{-2 / 3}$ from Eq. (35). For further discussions on the single-single population and this distribution see [114].

Finally, we now consider the relative normalizations of the single-single and binary-single distributions. As seen, for our assumed value of $F_{\mathrm{bs}}=0.01$, the 'sin-sin (uniform)' follows very closely the '3-body' distribution at high values of $f_{\mathrm{GW}}$. This is exactly what we derived in Eq. (22), in which we argued that the rates should be similar for encounters with $r_{\mathrm{p}}<R$, i.e. for encounters with $f_{\mathrm{GW}}>f_{\mathrm{GW}}(R)$, when the binary fraction is at the percent level. This, combined with the $\propto f_{\mathrm{GW}}^{-2 / 3}$ dependence, means that they must overlap at high $f_{\mathrm{GW}}$. The normalizations of the 'sin-sin (Plummer)' and ' 3 -body' distributions, over all $f_{\mathrm{GW}}$, derived using our analytical methods in Eq. (33), are also very similar. To conclude, our analytical and numerical methods agree fully on how and where the different BBH merger populations distribute in $f_{\mathrm{GW}}$ space.

\section{Orbital Eccentricity}

We now consider Fig. 4, which shows the orbital eccentricity distributions of the merging BBHs from Fig. 3 at 1 $\mathrm{Hz}$ and $10 \mathrm{~Hz}$. The $1 \mathrm{~Hz}$ regime is relevant for planned de- tectors such as DECIGO and Tian Qin, where the $10 \mathrm{~Hz}$ regime is relevant for currently operating LIGO/VIRGO, and future ground-based observatories such as the ET and $\mathrm{CE}$ (the ET and $\mathrm{CE}$ are likely to be sensitive at even lower GW frequencies).

Starting with the distribution at $1 \mathrm{~Hz}$ (top plot), we see that the single-single population (we here only show results for the Plummer's sphere) clearly dominates the distribution for all eccentricities $\log (e)>-1.5$. That is, in our model, eccentric deci-Hertz sources are likely to originate from single-single GW captures. This was also noticed in [105]; however, we have here been able to derive the correct normalization and how it relates to the 2-body and 3-body mergers. Note here, that we have in our model not included binary-binary [77] and any weak encounter driven mergers [68], which in principle also could contribute to eccentric deci-Hertz sources.

If we now consider the distribution at $10 \mathrm{~Hz}$ (bottom plot), we see that the single-single GW capture mergers don't contribute much to the population with an eccentricity resolvable by LIGO/VIRGO $(e>0.1)$. Instead, the single-single GW captures seem to fully dominate the region near $-2.5<\log (e)-1.5$, which interestingly could possibly be resolved by future detectors such as the ET/CE [e.g. 118]. Therefore, to get a complete picture of what future detectors might observe and how we can use it to constrain the origin of $\mathrm{BBH}$ mergers, the inclusion of single-single GW capture mergers seems to be very important.

Finally, because highly eccentric orbits will emit qausiperiodic burst of GWs at pericenter, it is observational relevant to consider the timescale between bursts for the single-single and 3-body mergers. As the time between bursts is equal to the $\mathrm{BBH}$ orbital time, $T_{\text {orb }}$, the period of interest is simply given by,

$$
T_{\mathrm{orb}}=\frac{2}{f_{\mathrm{GW}}}(1-e)^{-3 / 2},
$$

where we have used the gravitational wave peak frequency, $f_{\mathrm{GW}}$ (Eq. (35)), together with Kepler's law $T_{\text {orb }}=2 \pi \sqrt{a^{3} / 2 G m}$. In the high eccentricity limit, i.e. for $e \sim 1$ where bursts are relevant, it is useful to express the eccentricity as $e=1-10^{-x}$, from which $T_{\text {orb }}$ can be written as $T_{\mathrm{orb}}=\left(2 / f_{\mathrm{GW}}\right) 10^{3 x / 2}$. In the low eccentricity limit, i.e. for $e \ll 1$, the orbital period $T_{\text {orb }}$ is to leading order given by $T_{\text {orb }} \approx\left(2 / f_{\mathrm{GW}}\right)(1+3 e / 2)$.

For representative single-single GW capture mergers that have circularized to the point where they are detectable (near the end of the vertical rise of the grey and red tracks in Figure 5), but still on eccentric orbits, e.g., $e \sim 0.9$ and $f_{\mathrm{GW}}=0.1 \mathrm{~Hz}, T_{\text {orb }, \mathrm{ss}} \approx 10$ minutes. For the 3 -body capture mergers, in a similar regime, e.g., $e \sim 0.9$ and $f_{\mathrm{GW}}=10^{0.3} \mathrm{~Hz}, T_{\mathrm{orb}, \mathrm{bs}} \approx 30$ seconds. Work is currently being done on how to detect signals from eccentric burst-like sources [e.g. 120]. 


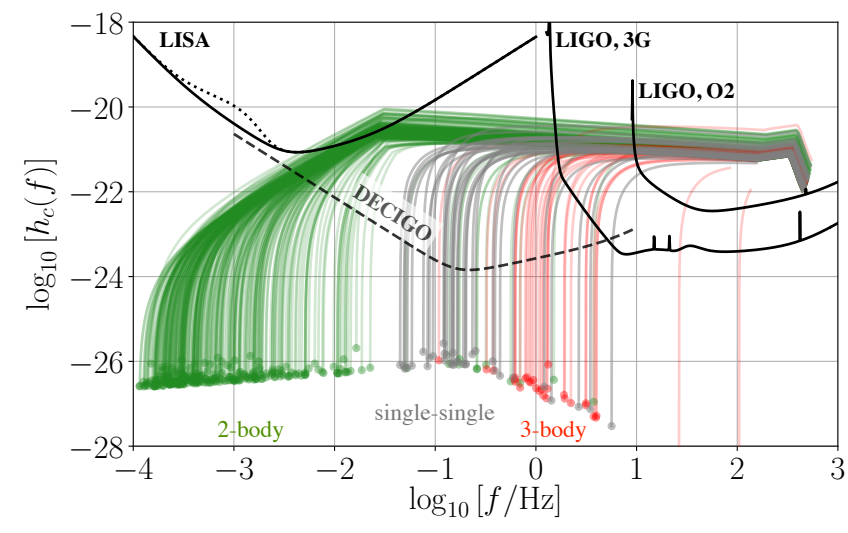

FIG. 5. Illustration of how our considered 2-body mergers (green), single-single GW capture mergers (grey), and 3-body mergers (red) distribute and evolve as a function of GW peak frequency (x-axis) and GW strain (y-axis). The shown data is a down-sampled version of the data used for Fig. 3. For the above figure we have assumed the sources distribute uniformly in volume up to a redshift $z=0.3$. On the figure is also shown sensitivity curves for LISA, DECIGO, LIGO 3G (the shown curve is for the ET), and LIGO O2 (current LIGO sensitivity). As seen, the single-single GW capture mergers fill out the gap between the 2-body and 3-body mergers, where DECIGO is most sensitive. The relative contributions from the three plotted merger types change with redshift and cluster parameters, therefore, future multi-band GW detectors have the potential to reveal exactly how and if BBHs are driven to merger in dense stellar clusters.

\section{Multi-band $G W$ Observations}

We end this section by showing how the 2-body mergers, single-single GW capture mergers, and 3-body mergers distribute and evolve as a function of GW peak frequency and GW strain to complement recent studies [e.g. 65, 66]. Results are shown in Fig. 5, where we have assumed a uniform source population up to a redshift of $z=0.3$, sampled from the data shown in Fig. 3 . On the figure is also shown sensitivity curves for LISA, DECIGO, LIGO 3G (ET), and LIGO O2 (current operational mode). As is clear from this figure, the singlesingle GW capture mergers form where DECIGO is most sensitive, and a future joint multi-band GW network, including instruments like LISA, DECIGO/Tian Qin, and $\mathrm{ET} / \mathrm{CE}$, will therefore be able to put tight constraints on exactly how BHs are brought to merger in dense clusters [e.g. 121].

Note here that the tracks shown in Fig. 5 show a representative characteristic strain at any point in the binary lifetime. They are only corrected for the observation-time or life-time of the detector in the sense that the characteristic strain is computed differently for binaries that will not significantly evolve in frequency over a chosen fouryear observation time. This results in the 'knee' in the 2body tracks (green) that is described more thoroughly in [65]. Binaries for which observation begins anywhere on the track to the left of the knee will not merge within an observation time. These tracks therefore serve more like an illustrative overview along side with Fig. 1 from [66]. However, we will provide a more in-depth signal-to-noise $(\mathrm{S} / \mathrm{N})$ analysis in an upcoming paper (for a discussion on multi-band S/N calculations see [e.g. 65, 121]), together with how the observational prospects change with cluster properties and $\mathrm{BH}$ masses.

\section{CONCLUSIONS}

Recent work on the formation of BBH mergers in GCs has shown that the inclusion of $\mathrm{PN}$ few-body dynamics leads to distinctive merger populations with measurable orbital eccentricities in both LISA and LIGO [e.g. 64-66, 78]. For these studies, the dynamical few-body channels that so far have been systematically explored include strong- and weak binary-single interactions [e.g. $68,70,75]$, and strong binary-binary interactions [77].

In this paper we have expanded on this emerging picture of how BBHs are driven to merger in GCs, by studying the formation of single-single GW capture mergers. For this we have focused on deriving the rate of $\mathrm{BBH}$ mergers from this single-single channel relative to that of BBH mergers forming during binary-single interactions. This approach gives us a much better handle on exactly how frequent single-single GW capture mergers might be, in contrast to other studies that report (highly uncertain) absolute rates.

Using analytical arguments, we find that the contribution from single-single GW capture mergers relative to those forming during binary-single interactions, does not strongly depend on either the absolute value of the central velocity dispersion, or the $\mathrm{BH}$ number density (see e.g. Eq. (19)). Instead, it mainly depends on the number of $\mathrm{BBHs}$ relative to single $\mathrm{BHs}$, i.e. the $\mathrm{BH}$ binary fraction, and the shape of the density profile of the single $\mathrm{BH}$ population. Assuming the single BHs follow either a uniform- or a Plummer's distribution, we have shown that the rate of single-single GW capture mergers should be comparable to that of mergers forming during binary-single interactions when the binary fraction is at the percent level. Interestingly, recent MC simulations (see Fig. 2) do in fact indicate that the fraction of dynamically formed BBHs is at the percent level, which leads us to conclude that single-single GW capture mergers are expected to form at an observable rate. Knowing the relative contributions from both single-single and binarysingle interactions is extremely important, as their interplay and resulting GW observables provide the key to probe how BBHs might form in dense stellar clusters.

Finally, using a semi-analytical MC framework we derived GW peak frequency- and eccentricity distributions for single-single GW capture mergers, and mergers forming during and in-between strong binary-single interactions. As also noticed by [105], the single-single GW capture mergers form right in the deci-Hertz regime where 
the proposed DECIGO and Tian Qin detectors are sensitive. For a binary fraction of $F_{\mathrm{bs}}=0.01$ and assuming a Plummer's sphere for the single BH population, we further illustrated that $\mathrm{BBHs}$ with eccentricities $\log (e)>-1.5$ at $1 \mathrm{~Hz}$ and $-1.5>\log (e)>-2.5$ at 10 $\mathrm{Hz}$ are dominated by the single-single GW capture channel. This has major implications for mapping out how BBHs form as a function of redshift in dense stellar clusters with DECIGO/Tian Qin as well as third generation $\mathrm{GW}$ detectors such as the ET and CE.

Acknowledgments. - It is a pleasure to thank the organizers of the CIERA Mini-workshop on PostNewtonian Dynamics in Stellar Clusters, December 5-8, 2018, where part of this work was initiated. The au- thors also thank Mirek Giersz for useful comments on the manuscript. JS acknowledges support from the Lyman Spitzer Fellowship and the European Unions Horizon 2020 research and innovation programme under the Marie Sklodowska-Curie grant agreement No. 844629. DJD acknowledges financial support from NASA through Einstein Postdoctoral Fellowship award number PF6170151. KK acknowledges support by the National Science Foundation Graduate Research Fellowship Program under Grant No. DGE-1324585. CR acknowledges supported from the Pappalardo Postdoctoral Fellowship at MIT. AA is supported by the Carl Tryggers Foundation through the grant CTS 17:113.
[1] B. P. Abbott et al., Physical Review Letters 116, 061102 (2016), 1602.03837.

[2] B. P. Abbott et al., Physical Review Letters 116, 241103 (2016), 1606.04855.

[3] B. P. Abbott et al., Physical Review X 6, 041015 (2016), 1606.04856.

[4] B. P. Abbott et al., Physical Review Letters 118, 221101 (2017), 1706.01812.

[5] B. P. Abbott et al., Physical Review Letters 119, 141101 (2017), 1709.09660.

[6] B. Zackay, T. Venumadhav, L. Dai, J. Roulet, and M. Zaldarriaga, arXiv e-prints , arXiv:1902.10331 (2019), 1902.10331.

[7] T. Venumadhav, B. Zackay, J. Roulet, L. Dai, and M. Zaldarriaga, arXiv e-prints , arXiv:1904.07214 (2019), 1904.07214.

[8]

[9] B. P. Abbott et al., Physical Review Letters 119, 161101 (2017), 1710.05832.

[10] M. Dominik et al., Astrophys. J.759, 52 (2012), 1202.4901

[11] M. Dominik et al., Astrophys. J.779, 72 (2013), 1308.1546 .

[12] M. Dominik et al., Astrophys. J.806, 263 (2015), 1405.7016 .

[13] K. Belczynski et al., Astrophys. J.819, 108 (2016), 1510.04615 .

[14] K. Belczynski, D. E. Holz, T. Bulik, and R. O'Shaughnessy, Nature (London)534, 512 (2016), 1602.04531 .

[15] K. Silsbee and S. Tremaine, Astrophys. J.836, 39 (2017), 1608.07642.

[16] A. Murguia-Berthier, M. MacLeod, E. Ramirez-Ruiz, A. Antoni, and P. Macias, Astrophys. J.845, 173 (2017), 1705.04698.

[17] C. L. Rodriguez and F. Antonini, Astrophys. J.863, 7 (2018), 1805.08212.

[18] S. L. Schrøder, A. Batta, and E. Ramirez-Ruiz, Astrophys. J. Lett. 862, L3 (2018), 1805.01269.

[19] N. Giacobbo and M. Mapelli, Mon. Not. Roy. Astron. Soc. 480, 2011 (2018), 1806.00001.

[20] A. S. Hamers and T. A. Thompson, arXiv e-prints , arXiv:1907.08297 (2019), 1907.08297.

[21] M. Spera et al., Mon. Not. Roy. Astron. Soc. 485, 889
(2019), 1809.04605.

[22] M. Mapelli, N. Giacobbo, F. Santoliquido, and M. C. Artale, Mon. Not. Roy. Astron. Soc. 487, 2 (2019), 1902.01419 .

[23] J. J. Eldridge, E. R. Stanway, and P. N. Tang, Mon. Not. Roy. Astron. Soc. 482, 870 (2019), 1807.07659.

[24] S. F. Portegies Zwart and S. L. W. McMillan, Astrophys. J.528, L17 (2000).

[25] S. Banerjee, H. Baumgardt, and P. Kroupa, Mon. Not. Roy. Astron. Soc. 402, 371 (2010), 0910.3954.

[26] A. Tanikawa, Mon. Not. Roy. Astron. Soc. 435, 1358 (2013), 1307.6268.

[27] Y.-B. Bae, C. Kim, and H. M. Lee, Mon. Not. Roy. Astron. Soc. 440, 2714 (2014), 1308.1641.

[28] C. L. Rodriguez et al., Physical Review Letters 115, 051101 (2015), 1505.00792.

[29] C. L. Rodriguez, S. Chatterjee, and F. A. Rasio, Phys. Rev. D93, 084029 (2016), 1602.02444.

[30] C. L. Rodriguez, C.-J. Haster, S. Chatterjee, V. Kalogera, and F. A. Rasio, Astrophys. J. Lett. 824, L8 (2016), 1604.04254.

[31] A. Askar, M. Szkudlarek, D. Gondek-Rosińska, M. Giersz, and T. Bulik, Mon. Not. Roy. Astron. Soc. 464, L36 (2017), 1608.02520.

[32] D. Park, C. Kim, H. M. Lee, Y.-B. Bae, and K. Belczynski, Mon. Not. Roy. Astron. Soc. 469, 4665 (2017), 1703.01568 .

[33] J. Hong et al., Mon. Not. Roy. Astron. Soc. 480, 5645 (2018), 1808.04514.

[34] U. N. Di Carlo et al., Mon. Not. Roy. Astron. Soc. 487, 2947 (2019), 1901.00863.

[35] N. Choksi, M. Volonteri, M. Colpi, O. Y. Gnedin, and H. Li, Astrophys. J.873, 100 (2019), 1809.01164.

[36] J. Kumamoto, M. S. Fujii, and A. Tanikawa, Mon. Not. Roy. Astron. Soc. 486, 3942 (2019), 1811.06726.

[37] F. Antonini and M. Gieles, arXiv e-prints , arXiv:1906.11855 (2019), 1906.11855.

[38] I. Bartos, B. Kocsis, Z. Haiman, and S. Márka, Astrophys. J.835, 165 (2017), 1602.03831.

[39] N. C. Stone, B. D. Metzger, and Z. Haiman, Mon. Not. Roy. Astron. Soc. 464, 946 (2017), 1602.04226.

[40] B. McKernan et al., ArXiv e-prints (2017), 1702.07818.

[41] R. M. O'Leary, B. Kocsis, and A. Loeb, Mon. Not. Roy. Astron. Soc. 395, 2127 (2009), 0807.2638. 
[42] J. Hong and H. M. Lee, Mon. Not. Roy. Astron. Soc. 448, 754 (2015), 1501.02717.

[43] J. H. VanLandingham, M. C. Miller, D. P. Hamilton, and D. C. Richardson, Astrophys. J.828, 77 (2016), 1604.04948.

[44] F. Antonini and F. A. Rasio, Astrophys. J.831, 187 (2016), 1606.04889.

[45] A. P. Stephan et al., Mon. Not. Roy. Astron. Soc. 460, 3494 (2016), 1603.02709.

[46] B.-M. Hoang, S. Naoz, B. Kocsis, F. A. Rasio, and F. Dosopoulou, ArXiv e-prints (2017), 1706.09896.

[47] A. S. Hamers, B. Bar-Or, C. Petrovich, and F. Antonini, Astrophys. J.865, 2 (2018), 1805.10313.

[48] A. Loeb, Astrophys. J. Lett. 819, L21 (2016), 1602.04735 .

[49] S. E. Woosley, Astrophys. J. Lett. 824, L10 (2016), 1603.00511.

[50] A. Janiuk, M. Bejger, S. Charzyński, and P. Sukova, ArXiv e-prints 51, 7 (2017), 1604.07132.

[51] D. J. D'Orazio and A. Loeb, Phys. Rev. D97, 083008 (2018), 1706.04211.

[52] S. Bird et al., Physical Review Letters 116, 201301 (2016), 1603.00464.

[53] I. Cholis et al., Phys. Rev. D94, 084013 (2016), 1606.07437.

[54] M. Sasaki, T. Suyama, T. Tanaka, and S. Yokoyama, Physical Review Letters 117, 061101 (2016), 1603.08338.

[55] B. Carr, F. Kühnel, and M. Sandstad, Phys. Rev. D94, 083504 (2016), 1607.06077.

[56] C. L. Rodriguez, M. Zevin, C. Pankow, V. Kalogera, and F. A. Rasio, Astrophys. J. Lett. 832, L2 (2016), 1609.05916.

[57] K. K. Y. Ng et al., Phys. Rev. D98, 083007 (2018), 1805.03046 .

[58] E. A. Huerta et al., Phys. Rev. D95, 024038 (2017), 1609.05933.

[59] L. Gondán, B. Kocsis, P. Raffai, and Z. Frei, Astrophys. J.855, 34 (2018), 1705.10781.

[60] E. A. Huerta et al., Phys. Rev. D97, 024031 (2018), 1711.06276.

[61] Y. Meiron, B. Kocsis, and A. Loeb, Astrophys. J.834, 200 (2017), 1604.02148.

[62] L. Randall and Z.-Z. Xianyu, arXiv e-prints , arXiv:1805.05335 (2018), 1805.05335.

[63] W. M. Farr et al., ArXiv e-prints (2017), 1706.01385.

[64] J. Samsing and D. J. D'Orazio, Mon. Not. Roy. Astron. Soc. (2018), 1804.06519.

[65] D. J. D'Orazio and J. Samsing, Mon. Not. Roy. Astron. Soc. 481, 4775 (2018), 1805.06194.

[66] K. Kremer et al., Phys. Rev. D99, 063003 (2019), 1811.11812.

[67] J. Samsing and D. J. D'Orazio, Phys. Rev. D99, 063006 (2019), 1807.08864.

[68] J. Samsing, A. S. Hamers, and J. G. Tyles, arXiv eprints , arXiv:1906.07189 (2019), 1906.07189.

[69] K. Gültekin, M. C. Miller, and D. P. Hamilton, Astrophys. J.640, 156 (2006).

[70] J. Samsing, M. MacLeod, and E. Ramirez-Ruiz, Astrophys. J.784, 71 (2014), 1308.2964.

[71] J. Samsing and E. Ramirez-Ruiz, Astrophys. J. Lett. 840, L14 (2017), 1703.09703.

[72] J. Samsing and T. Ilan, Mon. Not. Roy. Astron. Soc. 476, 1548 (2018), 1706.04672.
[73] J. Samsing, M. MacLeod, and E. Ramirez-Ruiz, Astrophys. J.853, 140 (2018), 1706.03776.

[74] J. Samsing and T. Ilan, Mon. Not. Roy. Astron. Soc. 482, 30 (2019), 1709.01660.

[75] J. Samsing, Phys. Rev. D97, 103014 (2018), 1711.07452.

[76] J. Samsing, A. Askar, and M. Giersz, Astrophys. J.855, 124 (2018), 1712.06186.

[77] M. Zevin, J. Samsing, C. Rodriguez, C.-J. Haster, and E. Ramirez-Ruiz, Astrophys. J.871, 91 (2019), 1810.00901.

[78] C. L. Rodriguez et al., Phys. Rev. D98, 123005 (2018), 1811.04926.

[79] T. A. Thompson, Astrophys. J.741, 82 (2011).

[80] L. Randall and Z.-Z. Xianyu, Astrophys. J.864, 134 (2018), 1802.05718.

[81] G. Fragione, E. Grishin, N. W. C. Leigh, H. B. Perets, and R. Perna, arXiv e-prints , arXiv:1811.10627 (2018), 1811.10627.

[82] L. Randall and Z.-Z. Xianyu, arXiv e-prints , arXiv:1902.08604 (2019), 1902.08604.

[83] G. Fragione, N. Leigh, and R. Perna, arXiv e-prints , arXiv:1903.09160 (2019), 1903.09160.

[84] G. Fragione and A. Loeb, Mon. Not. Roy. Astron. Soc. 486, 4443 (2019), 1903.10511.

[85] G. Fragione and O. Bromberg, arXiv e-prints , arXiv:1903.09659 (2019), 1903.09659.

[86] G. Fragione and B. Kocsis, Mon. Not. Roy. Astron. Soc. 486, 4781 (2019), 1903.03112.

[87] B. Kocsis and J. Levin, Phys. Rev. D 85, 123005 (2012).

[88] L. Gondán, B. Kocsis, P. Raffai, and Z. Frei, Astrophys. J.860, 5 (2018), 1711.09989.

[89] J. Lopez, Martin, A. Batta, E. Ramirez-Ruiz, I. Martinez, and J. Samsing, Astrophys. J.877, 56 (2019), 1812.01118 .

[90] J. Samsing et al., arXiv e-prints , arXiv:1901.02889 (2019), 1901.02889.

[91] K. Kremer, W. Lu, C. L. Rodriguez, M. Lachat, and F. Rasio, arXiv e-prints , arXiv:1904.06353 (2019), 1904.06353 .

[92] L. Blanchet, Living Reviews in Relativity 17 (2014), 1310.1528 .

[93] J. Samsing, M. MacLeod, and E. Ramirez-Ruiz, Astrophys. J.846, 36 (2017), 1609.09114.

[94] J. Samsing, N. W. C. Leigh, and A. A. Trani, Mon. Not. Roy. Astron. Soc. 481, 5436 (2018), 1803.08215.

[95] A. S. Hamers and J. Samsing, arXiv e-prints , arXiv:1904.09624 (2019), 1904.09624.

[96] A. S. Hamers and J. Samsing, arXiv e-prints , arXiv:1906.08666 (2019), 1906.08666.

[97] C. Hamilton and R. R. Rafikov, arXiv e-prints , arXiv:1902.01344 (2019), 1902.01344.

[98] C. Hamilton and R. R. Rafikov, arXiv e-prints , arXiv:1902.01345 (2019), 1902.01345.

[99] C. Hamilton and R. R. Rafikov, arXiv e-prints , arXiv:1907.00994 (2019), 1907.00994.

[100] S. Hild et al., Classical and Quantum Gravity 28, 094013 (2011), 1012.0908.

[101] B. P. Abbott et al., Classical and Quantum Gravity 34, 044001 (2017), 1607.08697.

[102] R. Hansen, Phys. Rev. D 5, 1021 (1972).

[103] M. H. Lee, Astrophys. J.418, 147 (1993).

[104] A. Rasskazov and B. Kocsis, Astrophys. J.881, 20 (2019), 1902.03242.

[105] X. Chen and P. Amaro-Seoane, Astrophys. J. Lett. 842, 
L2 (2017), 1702.08479.

[106] A. Askar, A. Askar, M. Pasquato, and M. Giersz, ArXiv e-prints (2018), 1811.06473.

[107] A. Askar, M. Arca Sedda, and M. Giersz, Mon. Not. Roy. Astron. Soc. 478, 1844 (2018), 1802.05284.

[108] S. Kawamura et al., Classical and Quantum Gravity 28, 094011 (2011).

[109] S. Isoyama, H. Nakano, and T. Nakamura, ArXiv eprints (2018), 1802.06977.

[110] J. Luo et al., Classical and Quantum Gravity 33, 035010 (2016), 1512.02076.

[111] M. Morscher, B. Pattabiraman, C. Rodriguez, F. A. Rasio, and S. Umbreit, Astrophys. J.800, 9 (2015), 1409.0866.

[112] D. C. Heggie, Mon. Not. Roy. Astron. Soc. 173, 729 (1975).
[113] A. M. Geller and N. W. C. Leigh, Astrophys. J. Lett. 808, L25 (2015), 1506.08830.

[114] B. Kocsis, M. E. Gáspár, and S. Márka, Astrophys. J.648, 411 (2006), astro-ph/0603441.

[115] C. L. Rodriguez and A. Loeb, Astrophys. J. Lett. 866, L5 (2018), 1809.01152.

[116] L. J. Spitzer, Astrophys. J.158, L139 (1969).

[117] H. C. Plummer, Mon. Not. Roy. Astron. Soc. 71, 460 (1911).

[118] B. Sun, Z. Cao, Y. Wang, and H.-C. Yeh, Phys. Rev. D92, 044034 (2015).

[119] L. Wen, Astrophys. J.598, 419 (2003).

[120] N. Loutrel and N. Yunes, Classical and Quantum Gravity 34, 135011 (2017), 1702.01818.

[121] A. Sesana, Physical Review Letters 116, 231102 (2016), 1602.06951 . 